\title{
Contractual Agreements for Coordination and Vendor-Managed Delivery under Explicit Transportation Considerations*
}

\author{
Ayşegül Toptal, ${ }^{1}$ Sıla Çetinkaya ${ }^{2}$ \\ ${ }^{1}$ Industrial Engineering Department, Bilkent University, Ankara, 06800, Turkey \\ ${ }^{2}$ Industrial and Systems Engineering Department, Texas A\&M University, \\ College Station, TX 77843-3131, USA
}

Received 22 August 2004; revised 9 December 2005; accepted 7 February 2006

DOI 10.1002/nav.20151

Published online 22 March 2006 in Wiley InterScience (www.interscience.wiley.com).

\begin{abstract}
We consider the coordination problem between a vendor and a buyer operating under generalized replenishment costs that include fixed costs as well as stepwise freight costs. We study the stochastic demand, single-period setting where the buyer must decide on the order quantity to satisfy random demand for a single item with a short product life cycle. The full order for the cycle is placed before the cycle begins and no additional orders are accepted by the vendor. Due to the nonrecurring nature of the problem, the vendor's replenishment quantity is determined by the buyer's order quantity. Consequently, by using an appropriate pricing schedule to influence the buyer's ordering behavior, there is an opportunity for the vendor to achieve substantial savings from transportation expenses, which are represented in the generalized replenishment cost function.

For the problem of interest, we prove that the vendor's expected profit is not increasing in buyer's order quantity. Therefore, unlike the earlier work in the area, it is not necessarily profitable for the vendor to encourage larger order quantities. Using this nontraditional result, we demonstrate that the concept of economies of scale may or may not work by identifying the cases where the vendor can increase his/her profits either by increasing or decreasing the buyer's order quantity. We prove useful properties of the expected profit functions in the centralized and decentralized models of the problem, and we utilize these properties to develop alternative incentive schemes for win-win solutions. Our analysis allows us to quantify the value of coordination and, hence, to identify additional opportunities for the vendor to improve his/her profits by potentially turning a nonprofitable transaction into a profitable one through the use of an appropriate tariff schedule or a vendor-managed delivery contract. We demonstrate that financial gain associated with these opportunities is truly tangible under a vendor-managed delivery arrangement that potentially improves the centralized solution.

Although we take the viewpoint of supply chain coordination and our goal is to provide insights about the effect of transportation considerations on the channel coordination objective and contractual agreements, the paper also contributes to the literature by analyzing and developing efficient approaches for solving the centralized problem with stepwise freight costs in the single-period setting. (c) 2006 Wiley Periodicals, Inc. Naval Research Logistics 53: 397-417, 2006
\end{abstract}

Keywords: channel coordination; contracts; vendor-managed delivery; vendor-managed inventory; integrated inventory/transportation decisions

\section{INTRODUCTION}

The fact that substantial savings can be realized due to coordination of the parties in the supply chain was recognized early in the 1970s [14,29], and since then buyer-vendor coordination has been a popular research area. Within the large spectrum of existing work in this area, centralized and decentralized models can be considered the two extremes. The

*This research was supported in part by NSF Grants CAREER/ DMII-0093654 and DMII-9908221.

Correspondence to: A. Toptal (toptal@bilkent.edu.tr);

S. Çetinkaya (sila@tamu.edu) traditional approach to coordination suggests integrating and modeling the replenishment decisions of the vendor and the buyer together. This approach qualifies as centralized modeling, and, undoubtedly, it provides the best result in terms of total system cost, i.e., the global optimum. In application, however, centralized control of the individual decisions of the buyer and the vendor may not be desirable, or feasible, even if both parties represent components of the same company. Furthermore, in real life, there is often a superior/subordinate relationship inherent in the situation where the dominant party prefers his/her priorities to lead the solution. As a result, decentralized modeling of the problem may be necessary. In a decentralized model, the parties solve 
their subproblems independently of each other with limited sharing of information.

While classical buyer-vendor coordination models can generally be characterized as falling into one of the abovementioned two modeling approaches, i.e., centralized vs. decentralized, the current trend is toward investigating ways to implement decentralized models without sacrificing too many of the cost saving benefits that result from centralized models. In keeping with this trend, the fundamental idea behind channel coordination is to identify the inefficiencies in decentralized solutions for the purpose of aligning the individual incentives for both parties with those of the centralized solutions. This requires the decentralized solution to be improved in a way that (i) it results in the same values for the decision variables as the centralized solution and (ii) it suggests a mutually agreeable way of sharing the resulting profits. The sharing can be done by means of quantity discounts, rebates, refunds, fixed payments between the parties, free delivery as in the case of a vendor-managed delivery (VMD) arrangement, or some combination of these. All of these methods for achieving centralized profits using a decentralized approach represent different forms of incentive schemes or so-called coordination mechanisms whose terms can be negotiable between the parties or are implicitly enforced by one party to influence the behavior of the other. As a result, the output of channel coordination, i.e., the so-called coordinated solution, combines the benefits of both centralized and decentralized solutions.

Despite the growing body of research on channel coordination, the existing literature overlooks important transportation considerations. In particular, the impact of truck/cargo capacity constraints and generalized inbound/outbound transportation cost functions are not taken into account. However, substantial system-wide efficiencies may be achievable by carefully incorporating such transportation considerations with the channel coordination objective. Recognizing a need for research on this topic, this paper is aimed at developing efficient coordination mechanisms for buyer-vendor problems with explicit transportation considerations represented via generalized replenishment costs. More specifically, the current paper extends the earlier work in channel coordination in order to consider: (i) truck capacity constraints for both inbound and outbound transport equipment and (ii) a general transportation cost structure that can explicitly represent the expenses associated with a fleet of vehicles, rather than a single truck. The paper seeks answers to the following key questions about channel coordination and contractual agreements under explicit transportation considerations.

Q1. What are the effects of transportation capacities and costs on the channel coordination objective, and how do we quantify these effects?
Q2. Can the classical coordination mechanisms, i.e., traditional pricing and incentive schemes, be used to achieve the channel coordination objective when the buyer-vendor system is subject to generalized transportation costs?

Q3. What are the benefits of alternative delivery agreements, such as a VMD contract, for the vendor and the buyer?

Q4. What insights can a third party transportation provider derive given the information that the buyervendor channel is coordinated?

For the purpose of providing realistic answers to the above questions, a practically common cost structure, which includes a fixed replenishment/delivery cost as well as a stepwise truck cost, is modeled for both the vendor and the buyer. This cost structure can be represented by the functional form

$$
C(Q)=K+\left\lceil\frac{Q}{P}\right\rceil R,
$$

where $Q$ denotes the replenishment quantity; $K$ denotes the fixed replenishment cost; $P$ denotes the truck capacity; and $R$ denotes the truck cost. As a result, the replenishment costs include both a fixed portion and a freight cost that is proportional to the number of trucks used. The functional form given in Expression (1) is also known in the literature as the multiple setup cost function, and it has several applications in batch industries where the manufacturer incurs a setup cost of $R$ for every batch of size $Q$ along with a production setup cost of $K$. Hence, the models in this paper are also applicable in a setting where the system replenishes via batch production. For several practical applications of the multiple setup cost function, see [1,21,24,35].

More specifically, considering a single-period, stochastic demand setting, we study two models. Model I is a special case where the generalized replenishment cost structure given by Expression (1) is incorporated into the vendor's costs only. Model II is a generalization of Model I, and it considers the generalized replenishment cost structure in Expression (1) for both the vendor and the buyer by making a distinction between the vendor's and buyer's fixed replenishment costs, i.e., $K_{v}$ and $K_{b}$; the vendor's and buyer's truck capacities, i.e., $P_{v}$ and $P_{b}$; and the buyer's and vendor's per truck costs, i.e., $R_{v}$ and $R_{b}$. However, it is important to note here that the insights gained in this paper are essentially based on the properties of the vendor's profit function which, in turn, depends on the vendor's costs. That is, in both models, the vendor's profit is not an increasing function of the buyer's order quantity because the generalized replenishment cost structure in Expression (1) is modeled for the vendor. Consequently, traditional coordination mechanisms, such as quantity discounts that exhibit economies of scale, are not always applicable for the problem under investigation. 
The coordinated solution in fact depends on both the centralized and the decentralized solutions: the former sets a benchmark for cost or profit whereas the latter helps to identify opportunities for coordination. Hence, for the problem of interest in this paper, we also analyze and compare the corresponding decentralized and centralized solutions. Although the focus of this paper is on coordination issues, the paper also contributes to the literature by analyzing and developing efficient solution approaches for solving the underlying centralized problem with stepwise costs in the single-period setting. Studying the analytical properties of the expected profit functions for the vendor and the buyer in the centralized and decentralized models of the problem, we find that quantity discounts that exhibit economies of scale are no longer sufficient under generalized replenishment costs. Hence, we develop alternative incentive schemes that are applicable when inefficiencies occur due to diseconomies of scale under explicit transportation considerations.

Based on our results, we identify opportunities for the vendor to improve his/her profits by potentially turning a nonprofitable transaction into a profitable one through the use of an appropriate tariff schedule or a VMD arrangement under which the vendor covers the buyer's transportation expenses. We argue that such a VMD arrangement may potentially improve on the centralized solution, and, hence, deliver a truly win-win alternative for the coordinated solution. We also quantify the financial gain associated with the proposed VMD arrangement and show that it is truly tangible for some problem instances. Finally, we provide insights for a third party transportation provider by showing that either the transportation considerations do not have an effect on the channel coordination objective (i.e., coordinated solutions with, and without, the generalized replenishment cost considerations are the same), or if they do have an affect, coordination is achieved at the full truck load level.

Next we revisit some basic ideas from the existing channel coordination literature, which provide a foundation for our analysis. This is followed in Section 3 by a summary of the literature where we also elaborate on the contributions of our study in relation to previous work. Notation and problem formulations are presented in Section 4. We discuss our mathematical results in Section 5 where we provide centralized and decentralized analyses of Models I and II and develop new pricing/incentive schemes allowing the vendor to influence the order quantity of the buyer. Section 6 presents a summary of some important managerial insights and concludes the paper.

\section{CHANNEL COORDINATION BASICS}

"Channel coordination" is a phrase coined in the marketing literature that applies to improving the total expected system profits in a decentralized model and to bringing them closer to those of a centralized model [33]. Concentrating on the stochastic demand, single-period setting that is of interest in this paper, let us give generic formulations of these two types of models.

More specifically, suppose that the buyer is a newsvendor and places an order of size $Q$ to the vendor, and the vendor reacts by fulfilling the buyer's order quantity. Under the centralized approach, $Q$ is specified by solving $\max _{Q \geq 0}\left[\Pi_{v}(Q)+\Pi_{b}(Q)\right]$ where $\Pi_{v}(Q)$ and $\Pi_{b}(Q)$ denote the vendor's and buyer's expected profit functions, respectively. In the rest of the paper, this problem is called the benchmark centralized model (BCM) whose optimal solution is denoted by $Q_{c}^{*}$ and referred as the centralized solution. For notational ease, we also let $\pi_{v}^{c}=\Pi_{v}\left(Q_{c}^{*}\right), \pi_{b}^{c}=\Pi_{b}\left(Q_{c}^{*}\right)$, and $\pi^{c}=\pi_{b}^{c}+\pi_{v}^{c}$. Consequently, $\pi_{v}^{c}$ and $\pi_{b}^{c}$ denote the vendor's and buyer's individual expected profits resulting from the centralized approach, respectively, whereas $\pi^{c}$ represents the optimal value of the total expected system profit under the centralized approach.

Under the decentralized approach, the buyer acts independently of the vendor, and, hence, $Q$ is specified by solving $\max _{Q \geq 0} \Pi_{b}(Q)$. In the rest of the paper, this problem is called the benchmark decentralized model (BDM) whose optimal solution is denoted by $Q_{d}^{*}$ and referred as the decentralized solution. In particular, under the decentralized approach considered in this paper, the vendor reacts to the buyer's order request passively and has no decision right in specifying the value of $Q$. Again, for notational ease, we let $\pi_{v}^{d}=\Pi_{v}\left(Q_{d}^{*}\right)$, $\pi_{b}^{d}=\Pi_{b}\left(Q_{d}^{*}\right)$, and $\pi^{d}=\pi_{b}^{d}+\pi_{v}^{d}$. Hence, $\pi_{v}^{d}$ and $\pi_{b}^{d}$ denote the vendor's and buyer's individual expected profits resulting from the decentralized approach, respectively, whereas $\pi^{d}$ represents the optimal value of the total expected system profit under the decentralized approach.

Since BCM maximizes the expected system profits, its objective function value is an upper bound on the total expected profits of the buyer-vendor system, i.e., $\pi^{d} \leq \pi^{c}$. In this sense, BCM can be used as a point of reference, and the gap between $\pi^{d}$ and $\pi^{c}$ can be considered an inducement to improve the outcome of the decentralized approach. For the problem under consideration, the centralized approach is only used for this theoretical purpose. Here, the underlying idea is that the centralized approach, under which the profits are shared in a judicious or arbitrary fashion, is not an agreeable practice by the parties. As a matter of fact, in the vein of the earlier papers from the channel coordination literature, cited in Section 3, we rely on this particular idea (stated in $\mathrm{O} 1$ below) as well as the following three additional observations.

O1. $\pi^{d} \leq \pi^{c}$ : The decentralized approach is inferior to the centralized approach as far as system profits are concerned. 
O2. $\pi_{b}^{d} \geq \pi_{b}^{c}$ : The buyer's expected profits under the decentralized solution are at least as great as those under the centralized solution.

O3. $\pi_{v}^{c} \geq \pi_{v}^{d}$ : The vendor's expected profits under the centralized solution are at least as great as those under the decentralized solution.

O4. $\pi_{v}^{c}-\pi_{v}^{d} \geq \pi_{b}^{d}-\pi_{b}^{c}$ : The vendor's gain from the centralized solution is no less than the buyer's loss from the decentralized solution.

We note that these observations are based on the decentralized approach discussed above where the buyer is the dominant party. Consequently, $\mathrm{O} 2$ follows because the buyer's objective can attain its maximum without consideration of any external constraints. Note that $\mathrm{O} 1$ and $\mathrm{O} 2$, together with the facts that $\pi^{c}=\pi_{b}^{c}+\pi_{v}^{c}$ and $\pi^{d}=\pi_{b}^{d}+\pi_{v}^{d}$, imply $\mathrm{O} 3$ and $\mathrm{O} 4$. It is worth noting that $\mathrm{O} 4$ is the key to the idea of channel coordination, because it suggests that one party's gain from the centralized solution is greater than the other party's loss. That is, the vendor's gain from using the centralized solution can be used to compensate the buyer's relative losses under the centralized solution as well as to increase the vendor's profits under the decentralized solution.

As we emphasize in Section 3, the previous literature concentrates on those cases where it is advantageous for the vendor to entice the buyer to increase his/her order quantity so that channel coordination can be achieved. The following proposition provides a sufficient condition under which it is, in fact, desirable for the vendor to induce the buyer to order more so that channel coordination can be achieved. Later in the paper, we identify cases where this condition does not hold, and, hence, it is not necessarily desirable for the vendor to receive larger orders.

PROPOSITION 1: If the vendor's expected profit is an increasing function of the buyer's order quantity, then the buyer's optimal order quantity in the BCM is no less than his/her optimal order quantity in the BDM.

PROOF: The proof is presented in Appendix A.1.

Although the above proposition sounds fairly comprehensive, there are many practical cases where the vendor's expected profits do not increase with the buyer's order quantity. One such practical situation is when the vendor replenishments incur costs as in Expression (1). For now, we proceed with a review of the related literature, but later, in Section 5, we demonstrate cases where $Q_{c}^{*}<Q_{d}^{*}$ so that the previously established coordination mechanisms are not workable.

\section{RELATED LITERATURE}

As we have already mentioned, despite the growing body of research on supply-chain coordination, no previous study in the literature investigates the effects of transportation considerations on the channel coordination objective. The existing work that specifically considers transportation issues focuses rather on the computation of jointly optimal lot-sizing policies, i.e., centralized solutions $[5,17,32]$. A common characteristic of these papers is that they concentrate on the case of deterministic demand, ignore channel coordination issues, and provide computational solution approaches. This is mainly because, when transportation considerations are modeled explicitly, the deterministic centralized problems alone are computationally challenging, and, hence, research on the topic is methodologically oriented.

In fact, a significant number of the earlier papers in channel coordination also consider the deterministic demand case and propose quantity discounts as a means to modify the behavior of the buyer so that the channel coordination objective can be achieved. One of the pioneering papers in this stream of research is by Monahan [26] who studies a single-vendor single-buyer problem where the vendor's replenishment lot size is equal to the buyer's order quantity per replenishment cycle. The buyer's replenishment problem is modeled using the classical EOQ framework; however, the inventory holding costs of the buyer are not incorporated into the model. The author shows that an all-unit discount schedule offered by the vendor to the buyer can increase the vendor's profits while putting the buyer in a "no worse" situation. In a later study, Banerjee [2] modifies Monahan's model to incorporate holding costs and a finite production rate for the vendor. A further generalization is studied by Lee and Rosenblatt [22] who assume that the replenishment lot size of the vendor is an integer multiple of the replenishment lot size of the buyer and, therefore, incorporate a constraint that limits the maximum value of the unit discount. Other notable extensions of the deterministic demand channel coordination problem $[3,16,18,20,34]$ consider (i) the case of multiple buyers where coordinating the channel in compliance with the Robinson Patman Act is a challenging research problem and (ii) the case of price-sensitive demand where channel coordination cannot be guaranteed by quantity discounts alone.

The earlier deterministic demand models we have cited so far consider quantity discounts and/or fixed payments as mechanisms for channel coordination. On the other hand, considering cases involving stochastic demand, recent work focuses on other alternative mechanisms such as buyback policies [12,27], return policies [30], and rebate policies [31]. These mechanisms, along with the quantity discounts and/or fixed payments proposed in the earlier papers that consider deterministic demand, rely on the idea of inducing the buyer to choose a larger order quantity than his/her optimal decentralized order quantity. On the other hand, to the best of our knowledge, no previous work explicitly analyzes the case of interest in this paper where, in some situations, it may not be desirable for the vendor to induce the buyer to order 
more. Highlighting such practical cases, our analyses provide a comparison of the centralized solutions with, and without, transportation considerations for both Models I and II. This comparison not only helps us to develop efficient solution approaches for the problem by obtaining bounds on the optimal replenishment quantities but also leads to insightful results for answering the four questions of interest listed in Section 1. These results are discussed throughout Section 5 as they are developed and summarized with our concluding comments in Section 6.

Finally, we note that all of the papers cited above, as well as the current paper, take the viewpoint of the vendor and assume that the vendor has full information about the buyer's parameters. In some recent studies, however, different aspects of channel coordination-such as the impact of asymmetric information, the value of information sharing, and alternative contractual settings that take the viewpoint of the buyerhave been investigated, e.g., see [4, 8, 13, 15, 23]. A similar investigation for the problem considered in the current paper remains an area of future research.

\section{NOTATION AND PROBLEM FORMULATION}

In the vein of the recent papers from the channel coordination literature, we consider a stylistic setting consisting of a vendor and a buyer who operate under the assumptions of the classical newsvendor problem to satisfy random demand for a single item with a short product life cycle. The full order for the cycle is placed before the cycle begins and no additional orders are accepted by the vendor. In this simple setting with stochastic demand, depending on the available supply (replenishment quantity) at the buyer, either one or the other of the following cases arises. If demand during the period exceeds the supply, then the buyer is out of stock and additional demand is lost, incurring a unit lost sale cost denoted by $b$. On the other hand, if demand during the period is less than the available supply at the buyer, then there are excess items at the buyer that can be sold at a unit salvage value denoted by $v$. The unit retail price at the buyer is denoted by $r$. The vendor simply orders or produces the buyer's required replenishment quantity. Since the general replenishment cost structure of the vendor can also be interpreted as a capacitated setup cost due to production at the vendor's site in response to the buyer's order, the results presented here are potentially applicable to the case where the vendor is a make-to-order manufacturer, i.e., lot-for-lot manufacturer. Also, the vendor incurs a unit purchase/production cost denoted by $p$ and charges a unit wholesale price denoted by $c$ where $v<p<c<r$. In addition to the fixed replenishment cost denoted by $K_{v}$, the vendor incurs a freight cost, given by $\left\lceil Q / P_{v}\right\rceil R_{v}$, for a replenishment quantity of $Q$ units, where $P_{v}$ is the truck capacity and $R_{v}$ is the per truck cost. As we noted earlier, in Model II, we incorporate a similar generalized replenishment cost structure for the buyer as well as the vendor. For this purpose, we denote the buyer's truck capacity and per truck cost by $P_{b}$ and $R_{b}$, respectively. The buyer's fixed cost of replenishment is denoted by $K_{b}$ in both Models I and II.

Next, we provide a summary of the notation used so far and introduce some new notation that will be used throughout the text.

$Q$ : Number of items ordered by the buyer.

$X$ : Random variable representing the buyer's total demand.

$f(\cdot)$ : Probability density function of demand.

$F(\cdot)$ : Probability distribution function of demand.

$p$ : Vendor's per unit procurement cost.

$c$ : Per unit wholesale price.

$r$ : Per unit retail price.

$v$ : Per unit salvage value at the buyer.

$b$ : Per unit lost sale cost at the buyer.

$R_{b}$ : Buyer's cost per truck.

$R_{v}$ : Vendor's cost per truck.

$P_{b}$ : Truck capacity for buyer's replenishment.

$P_{v}$ : Truck capacity for vendor's replenishment.

$\bar{\Pi}_{b}(Q)$ : Buyer's expected profit function excluding truck costs.

$\bar{\Pi}_{v}(Q)$ : Vendor's expected profit function excluding truck costs.

$\bar{\Pi}_{c}(Q): \quad$ Expected system profit function excluding truck costs, i.e., $\bar{\Pi}_{c}(Q)=\bar{\Pi}_{v}(Q)+\bar{\Pi}_{b}(Q)$.

$\Pi_{b}(Q)$ : Buyer's expected profit function with truck costs.

$\Pi_{v}(Q)$ : Vendor's expected profit function with truck costs.

$\Pi_{c}^{I}(Q)$ : Expected system profit function for Model I, i.e., $\Pi_{c}^{I}(Q)=\Pi_{v}(Q)+\bar{\Pi}_{b}(Q)$.

$\Pi_{c}^{I I}(Q)$ : Expected system profit function for Model II, i.e., $\Pi_{c}^{I I}(Q)=\Pi_{v}(Q)+\Pi_{b}(Q)$.

Using the notation defined above, we can write

$$
\begin{array}{r}
\bar{\Pi}_{b}(Q)=(-c+v) Q-K_{b}+(r-v) \int_{0}^{\infty} x f(x) d x \\
-(r-v+b) \int_{Q}^{\infty}(x-Q) f(x) d x .
\end{array}
$$

It can be easily shown that $\bar{\Pi}_{b}(Q)$ is a strictly concave function with a unique maximizer denoted by $\bar{Q}_{d}^{*}$ that satisfies

$$
F\left(\bar{Q}_{d}^{*}\right)=\frac{r+b-c}{r+b-v} .
$$

In fact, $\bar{Q}_{d}^{*}$ is the optimal value of the buyer's order quantity in the decentralized system if the stepwise truck costs for 
the buyer are ignored. When truck costs are excluded, the vendor's profits as a function of the buyer's order quantity, i.e., $\bar{\Pi}_{v}(Q)$, are given by $(c-p) Q-K_{v}$. It follows that

$$
\begin{array}{r}
\bar{\Pi}_{c}(Q)=(-p+v) Q-K_{b}-K_{v}+(r-v) \int_{0}^{\infty} x f(x) d x \\
-(r-v+b) \int_{Q}^{\infty}(x-Q) f(x) d x .
\end{array}
$$

The above function has the same form as $\bar{\Pi}_{b}(Q)$, given by Expression (2), and, thus, its unique maximizer $\bar{Q}_{c}^{*}$ satisfies

$$
F\left(\bar{Q}_{c}^{*}\right)=\frac{r+b-p}{r+b-v} .
$$

REMARK 1: The buyer's optimal order quantity in the centralized model without truck costs is at least as large as the one in the corresponding decentralized model, i.e., $\bar{Q}_{c}^{*} \geq \bar{Q}_{d}^{*}$.

The result stated in Remark 1 is a consequence of Proposition 1. That is, if the vendor does not have truck capacity, then his/her expected profit, i.e., $\bar{\Pi}_{v}(Q)$, is an increasing function of the buyer's order size, and, hence, the channel is coordinated using an increased order quantity.

Recall that in both Models I and II, the vendor has the generalized replenishment cost structure represented by Expression (1). Therefore, in both models, the vendor's expected profit function is given by

$$
\begin{aligned}
\Pi_{v}(Q)=\bar{\Pi}_{v}(Q)-\left\lceil\frac{Q}{P_{v}}\right\rceil R_{v} & \\
& =(c-p) Q-K_{v}-\left\lceil\frac{Q}{P_{v}}\right\rceil R_{v}
\end{aligned}
$$

On the other hand, the buyer's expected profit functions and, hence, buyer's subproblems, in Model I and Model II are different. Since truck capacity and costs are ignored for the buyer in Model I, the buyer's subproblem in this model is to maximize Expression (2). In Model II, however, the buyer wishes to maximize $\Pi_{b}(Q)=\bar{\Pi}_{b}(Q)-\left\lceil Q / P_{b}\right\rceil R_{b}$.

Under these assumptions, in both models, the problem is to decide on the replenishment quantity for the buyer-vendor system under consideration. Next, we discuss how to compute this quantity using the decentralized and centralized modeling approaches for Models I and II. By knowing the properties of the expected profit expressions in the buyer's and vendor's decentralized subproblems, it will be easier to solve the centralized problem where the sum of these two profit functions is maximized. Hence, we concentrate first on the decentralized approach.

\section{ANALYSIS OF THE PROBLEM}

We begin by presenting some important properties of the underlying profit functions that are common to both the decentralized and the centralized models of the problem. For this purpose, let us first consider

$$
h(Q)=g(Q)-\left\lceil\frac{Q}{P}\right\rceil R,
$$

where $g(Q)$ is a concave, continuous function defined over all nonnegative real values of $Q$. Define $q$ as the smallest maximizer of $g(Q)$. Observe that the second term of $h(Q)$ is a stepwise function. Denoting the smallest maximizer of $h(Q)$ by $Q^{*}$, we present a method for computing $Q^{*}$. The maximization procedure for $h(Q)$ will be useful for optimizing the decentralized and centralized objective functions for Models I and II. For this reason, we present the following properties of $h(Q)$ that allow us to simplify this procedure. We note that the proofs of Properties 1-3 and Proposition 2 are presented in Appendix A.2. Also, we define $l=\lceil q / P\rceil$ and let $Q_{1}$ and $Q_{2}$ denote two nonnegative numbers.

PROPERTY 1: We have $h(Q)<h(q), \forall Q>l P$. That is, the function value at $q$ is greater than the function values beyond $l P$.

Property 1 implies that $Q^{*} \in \mathcal{A}^{1}=\{Q: 0 \leq Q \leq l P\}$.

PROPERTY 2: Suppose that $Q_{1}<Q_{2} \leq q$. If $(k-1) P<$ $Q_{1}<Q_{2} \leq k P \leq q$ where $k$ is a positive integer; then $h\left(Q_{1}\right)<h\left(Q_{2}\right)$. That is, $h(Q)$ is piecewise increasing over $Q \leq q$.

It follows from Property 2 that if $(l-1) P<Q_{1}<Q_{2} \leq q$ then $h\left(Q_{1}\right)<h\left(Q_{2}\right)$. Hence, Properties 1 and 2 reduce the set within which we should look for the maximizers of $h(Q)$ to integer multiples of $P$ that are less than or equal to $q$ and to all reals between and including $q$ and $l P$. That is,

$$
\begin{aligned}
& Q^{*} \in \mathcal{A}^{2}=\{Q: Q=k P<q, \\
& \qquad k \in\{0,1,2, \ldots\} \text {, and } q \leq Q \leq l P\} .
\end{aligned}
$$

The next property reduces this set further.

PROPERTY 3: If there exists a nonnegative $Q_{1}$ such that $Q_{1}>q$ and $g\left(Q_{1}\right)<g(q)$, then over $Q>Q_{1}$, we have $g(Q)<g\left(Q_{1}\right)$, and, hence, $h(Q)<h\left(Q_{1}\right)$. In other words, for $Q>q$, if $g(Q)$ is decreasing (non-increasing) over a specific region then $h(Q)$ is also decreasing (non-increasing) over the same region.

Recall that $q$ is defined as the smallest maximizer of $g(Q)$. Hence, over $q \leq Q \leq l P$, there may be other $Q$ values such that $g(Q)=g(q)$. However, Property 3 implies that, for 


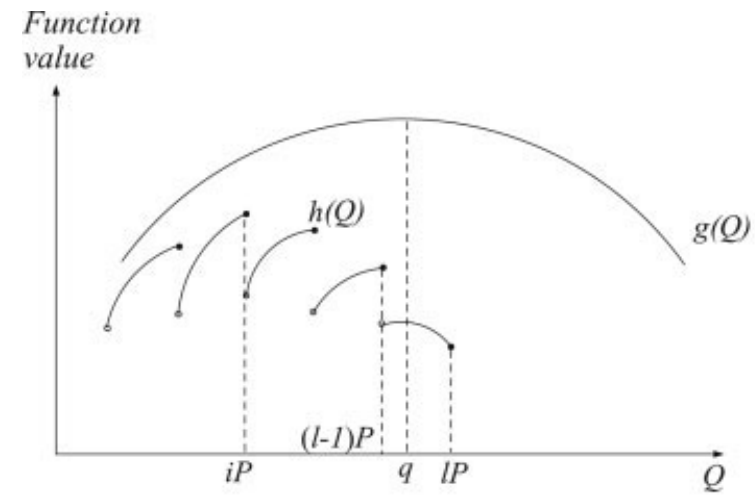

a. Case 1

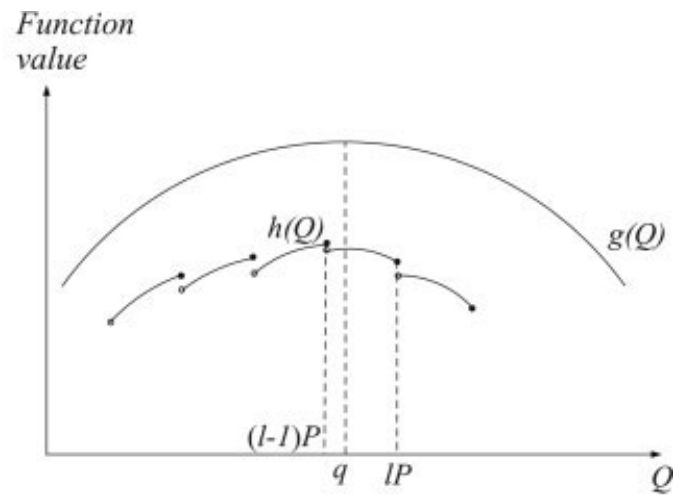

b. Case 2

Figure 1. Different illustrations of $h(Q)$ where Case 1 illustrates the case $\mathcal{F} \neq \emptyset$, and Case 2 illustrates the case $\mathcal{F}=\emptyset$.

the purpose of computing $Q^{*}$, we can ignore such $Q$ values. That is,

$$
Q^{*} \in \mathcal{A}^{3}=\{Q: Q=k P<q, k \in\{0,1,2, \ldots\} \text {, and } q\} .
$$

Let us define

$$
\begin{aligned}
\mathcal{F}=\{k \in\{0,1,2, \ldots\}: g((k+1) P-g(k P)) \leq R, \\
(k+1) P \leq q\} \quad \text { and } \quad i=\min \{k \text { s.t. } k \in \mathcal{F}\} .
\end{aligned}
$$

\section{PROPOSITION 2:}

$$
Q^{*}= \begin{cases}i P & \text { if } \mathcal{F} \neq \emptyset, \\ q & \text { if } \mathcal{F}=\emptyset \text { and } h(q)>h((l-1) P), \\ (l-1) P & \text { otherwise. }\end{cases}
$$

The above proposition indicates that the maximizer of $h(Q)$ in Expression (7) is either $q$, which is the maximizer of the concave component $g(Q)$, or an integer multiple of $P$ that is less than $q$ (see Fig. 1 for different illustrations of $h(Q)$ when $g(Q)$ is strictly concave). We use Proposition (2) to solve the centralized problem for Model I and the decentralized problem for Model II. Next, we present an analysis of the vendor's expected profit function. As we have mentioned earlier, when the generalized replenishment cost structure given by Expression (1) is modeled for the vendor, the coordination problem is interesting in that there are cases where the vendor favors a smaller order quantity from the buyer. The properties of the vendor's expected profit function are useful for characterizing these cases.

\subsection{Vendor's Profit Function: $\Pi_{v}(Q)$}

Recall Eq. (6), which gives an expression of the vendor's profit function $\Pi_{v}(Q)$. Figure 2 provides an illustration of $\Pi_{v}(Q)$ based on the following properties of this function. Property 4 is a direct result of Proposition 2 for the case

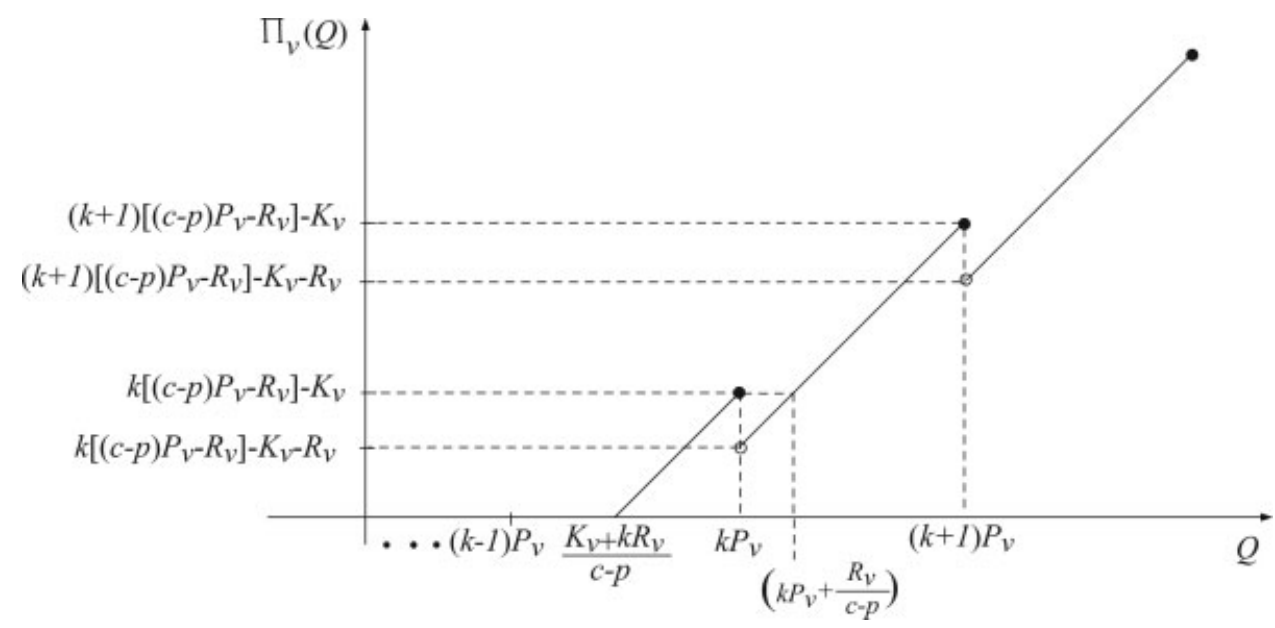

Figure 2. An illustration of $\Pi_{v}(Q)$ when $(c-p) P_{v}>R_{v}$. 


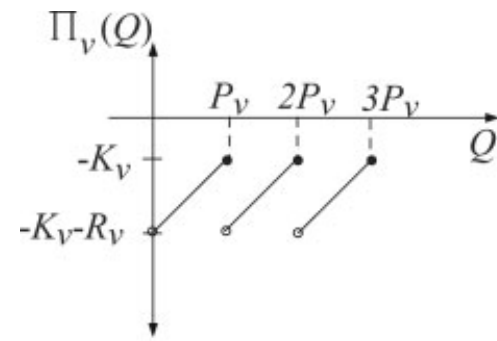

a. $(c-p) P_{v}=R_{v}$

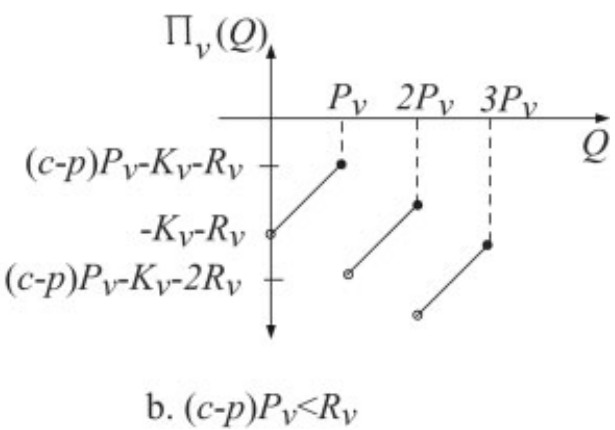

Figure 3. Different illustrations of $\Pi_{v}(Q)$.

$g(Q)=(c-p) Q-K_{v}$, i.e., $q=\infty$, and, hence, its proof is omitted. Proofs of Properties 5-7 are presented in Appendix A.3.

PROPERTY 4: $\Pi_{v}\left(Q_{2}\right)>\Pi_{v}\left(Q_{1}\right), \forall Q_{1}, Q_{2}$ s.t. $(k-1) P_{v}$ $<Q_{1}<Q_{2} \leq k P_{v}$ and $k \in Z^{+}$. In other words, $\Pi_{v}(Q)$ is piecewise increasing.

PROPERTY 5: If $(c-p) P_{v} \leq R_{v}$, then $\Pi_{v}(Q)<0, \forall Q \geq$ 0 , i.e., the vendor is at loss for any $Q$ (see Fig. 3). If ( $c-$ p) $P_{v}>R_{v}, \exists Q \geq 0$ s.t. $\Pi_{v}(Q)>0$.

The above property is important because it implies that when the vendor's revenue from sales of a full truck load of items (i.e., $P_{v}$ ) does not exceed the per truck cost, the vendor does not profit from this one-time transaction regardless of the order quantity. However, by coordinating the channel, the vendor still has the opportunity to decrease the magnitude of his/her losses (see Fig. 3). If the revenue from selling $P_{v}$ units exceeds the truck cost, the increase in the vendor's profits from the coordinated solution may even turn a nonprofitable transaction into a profitable one for the vendor.

PROPERTY 6: If $(c-p) P_{v}>R_{v}$, then $\Pi_{v}\left((k+1) P_{v}\right)>$ $\Pi_{v}\left(k P_{v}\right), \forall k$. That is, if $(c-p) P_{v}>R_{v}$, the vendor's profits at integer multiples of $P_{v}$ are increasing.

PROPERTY 7: If $(c-p) P_{v}>R_{v}$, then $\Pi_{v}\left(k P_{v}\right)=$ $\Pi_{v}\left(k P_{v}+R_{v} /(c-p)\right)>\Pi_{v}(Q), \forall Q$ s.t. $k P_{v}<Q<$ $k P_{v}+R_{v} /(c-p), k \in Z^{+}$.

In the above property, $R_{v} /(c-p)$ is the least number of items that should be sold by the vendor to cover the cost of an additional truck. As seen in Fig. 2, if the buyer's order quantity under the market price results in a truck with a load of less than $R_{v} /(c-p)$ units for the vendor, i.e., $k P_{v}<Q<$ $k P_{v}+R_{v} /(c-p)$, then the vendor can increase his/her profits either by increasing the order quantity or by decreasing it to the previous full truck load. The exact value of the order quantity in the coordinated solution, however, depends also on the buyer's expected profit function. This quantity is found using the centralized model, and it provides the maximum increase in system profits by balancing the increase in buyer's costs with the increase in vendor's profits.

Next, we derive the decentralized and centralized solutions for Model I, and we compare the order quantities in the two solutions to gain insights into coordinating the channel. We rely on the earlier analysis for maximization of function $h(Q)$ in Expression (7) for optimizing our models, and we use the properties of the vendor's profit function in simplifying our results.

\subsection{Model I}

\subsubsection{Decentralized Solution for Model I}

As described in Section 4, the buyer's decentralized decision problem in Model $\mathrm{I}$ is to find the value of $Q$ that maximizes $\bar{\Pi}_{b}(Q)$ given by Expression (2). Let $Q_{d, 1}^{*}$ denote the optimal solution of this problem. Obviously, $Q_{d, 1}^{*}=\bar{Q}_{d}^{*}$.

\subsubsection{Centralized Solution for Model I}

The objective function to be maximized in the centralized model is the sum of the expected vendor profits and expected buyer profits, which in turn is given by

$$
\Pi_{c}^{I}(Q)=\Pi_{v}(Q)+\bar{\Pi}_{b}(Q) .
$$

Noting that $\Pi_{v}(Q)=\bar{\Pi}_{v}(Q)-\left\lceil Q / P_{v}\right\rceil R_{v}$, this function can be expressed as

$$
\Pi_{c}^{I}(Q)=\bar{\Pi}_{b}(Q)+\bar{\Pi}_{v}(Q)-\left\lceil\frac{Q}{P_{v}}\right\rceil R_{v} .
$$

Using the fact that $\bar{\Pi}_{c}(Q)=\bar{\Pi}_{v}(Q)+\bar{\Pi}_{b}(Q)$, the above expression reduces to

$$
\Pi_{c}^{I}(Q)=\bar{\Pi}_{c}(Q)-\left\lceil\frac{Q}{P_{v}}\right\rceil R_{v} .
$$


Recall from Section 4 that $\bar{\Pi}_{c}(Q)$, given by Expression (4), is the expected system profits of the buyer-vendor system without truck capacity and costs. This is a strictly concave function whose maximizer $\bar{Q}_{c}^{*}$ is given by Expression (5). Denoting the optimum level of the buyer's order quantity in the centralized solution of Model I by $Q_{c, 1}^{*}$, we have the following property.

PROPERTY 8: The following are true for the objective function values of Model I:

1. $\Pi_{c}^{I}\left(Q_{c, 1}^{*}\right)<\bar{\Pi}_{c}\left(\bar{Q}_{c}^{*}\right)$;

2. If $\bar{\Pi}_{c}\left(\bar{Q}_{c}^{*}\right)<R_{v}$ then $\Pi_{c}^{I}\left(Q_{c, 1}^{*}\right)<0$.

PROOF: The proof is presented in Appendix A.4.

Note that $\Pi_{c}^{I}(Q)$ consists of a strictly concave function, i.e., $\bar{\Pi}_{c}(Q)$, and a stepwise term as function $h(Q)$ in Expression (7). Therefore, the maximizer can be computed using Proposition (2) by substituting $g(Q)=\bar{\Pi}_{c}(Q)$ and $q=\bar{Q}_{c}^{*}$ so that $h(Q)=\Pi_{c}^{I}(Q)$. As a result,

$$
Q_{c, 1}^{*}= \begin{cases}i P_{v} & \text { if } \mathcal{F} \neq \emptyset \\ \bar{Q}_{c}^{*} & \text { if } \mathcal{F}=\emptyset \text { and } \Pi_{c}^{I}\left(\bar{Q}_{c}^{*}\right) \\ & >\Pi_{c}^{I}\left(\left(\left\lceil\frac{\bar{Q}_{c}^{*}}{P_{v}}\right\rceil-1\right) P_{v}\right) \\ \left(\left\lceil\frac{\bar{Q}_{c}^{*}}{P_{v}}\right]-1\right) P_{v} & \text { otherwise. }\end{cases}
$$

where $\mathcal{F}=\left\{k \in Z^{+}:-\bar{\Pi}_{c}\left(k P_{v}\right)+\bar{\Pi}_{c}\left((k+1) P_{v}\right) \leq\right.$ $\left.R_{v},(k+1) P_{v} \leq \bar{Q}_{c}^{*}\right\}$ and $i=\min \{k$ s.t. $k \in \mathcal{F}\}$.

REMARK 2: It follows from Expression (11) that $Q_{c, 1}^{*} \leq$ $\bar{Q}_{c}^{*}$. That is, the centralized order quantity of the system, considering truck capacity and costs for the vendor, is at most as large as that of the system without considering truck capacity and costs. Furthermore, if $Q_{c, 1}^{*}$ is not equal to $\bar{Q}_{c}^{*}$, then it can only take a value that is an integer multiple of $P_{v}$.

Remark 2 provides an important insight into the dynamics of the buyer-vendor system. That is, if the vendor has a general replenishment cost structure as in Expression (1), then either the transportation constraints and costs do not have an effect on the coordinated buyer-vendor system or, if they do have an effect, they force the quantity to be a full truck load.

PROPERTY 9: Let $F(\cdot)$ and $f(\cdot)$ denote the distribution and density functions of the demand, respectively. We have

$$
\begin{aligned}
& F\left((k+1) P_{v}\right) \\
& \geq \frac{(r-p+b) P_{v}-R_{v}+(r-v+b) \int_{k P_{v}}^{(k+1) P_{v}}\left(x-k P_{v}\right) f(x) d x}{(r-v+b) P_{v}}, \\
& \forall k \in \mathcal{F}^{o}, \quad \text { (12) }
\end{aligned}
$$

where $\mathcal{F}^{o}=\left\{k:-\bar{\Pi}_{c}\left(k P_{v}\right)+\bar{\Pi}_{c}\left((k+1) P_{v}\right) \leq R_{v}\right\}$.

PROOF: The proof is presented in Appendix A.4.

Note that Inequality (12) is a more explicit representation of the constraint $-\bar{\Pi}_{c}\left(k P_{v}\right)+\bar{\Pi}_{c}\left((k+1) P_{v}\right) \leq R_{v}$ that is satisfied by $k \in \mathcal{F}^{o}$. If the minimum positive integer $k$ for which Inequality (12) holds also satisfies $(k+1) P_{v} \leq \bar{Q}_{c}^{*}$, then the $i$ value in Expression (11) is given by $k$. Therefore, for specific distribution and density functions, the representation given in Inequality (12) may lead to a close form expression for the value of $i$ in Expression (11). This representation also enables a nice interpretation: In Inequality (12), the expression in the numerator can be considered the system's cost associated with not ordering another full truck load of demand in addition to $k$ full trucks. This is similar to the underage cost of each unit demand that cannot be met. Similarly, $(p-v) P_{v}+R_{v}-(r-v+b) \int_{k P_{v}}^{(k+1) P_{v}}\left(x-k P_{v}\right) f(x) d x$ can be interpreted as the cost associated with ordering an additional full truck load in excess of $k$ full trucks. Hence, the denominator of Inequality (12) can be interpreted as the sum of overage and underage system costs associated with a truck load in addition to $k$ trucks.

Based on the vendor's cost parameters and the properties described in Section 5.1, there are some special cases where Expression (11) can be simplified further. Theorem 1 and Propositions 3 and 4 discuss such cases.

THEOREM 1: Suppose $(c-p) P_{v} \geq R_{v}$.

- If $Q_{d, 1}^{*} \neq\left\lceil Q_{d, 1}^{*} / P_{v}\right\rceil P_{v}$ (i.e., $Q_{d, 1}^{*}$ is not a full-truckload shipment), then $Q_{c, 1}^{*} \geq\left(\left\lceil Q_{d, 1}^{*} / P_{v}\right\rceil-1\right) P_{v}$.

- If $Q_{d, 1}^{*}=\left\lceil Q_{d, 1}^{*} / P_{v}\right\rceil P_{v}$, then $Q_{c, 1}^{*} \geq Q_{d, 1}^{*}$.

That is, $Q_{c, 1}^{*} \geq\left\lfloor Q_{d, 1}^{*} / P_{v}\right\rfloor P_{v}$.

PROOF: The proof is presented in Appendix A.4.

The above theorem simplifies the computation of $Q_{c, 1}^{*}$, given by Expression (11), in the following way. When $(c-p) P_{v} \geq R_{v}$, we do not need to consider certain values for $i$. That is, we compute $Q_{d, 1}^{*}$, and if $Q_{d, 1}^{*} \neq$ $\left\lceil Q_{d, 1}^{*} / P_{v}\right\rceil P_{v}$, then we construct $\mathcal{F}$ by checking the conditions $-\bar{\Pi}_{c}\left(k P_{v}\right)+\bar{\Pi}_{c}\left((k+1) P_{v}\right) \leq R_{v}$ and $(k+1) P_{v} \leq \bar{Q}_{c}^{*}$ for $k \geq\left(\left\lceil Q_{d, 1}^{*} / P_{v}\right\rceil-1\right)$. Therefore, $\left(\left\lceil Q_{d, 1}^{*} / P_{v}\right\rceil-1\right) P_{v} \leq$ $Q_{c, 1}^{*} \leq \bar{Q}_{c}^{*}$. On the other hand, if $Q_{d, 1}^{*}=\left\lceil Q_{d, 1}^{*} / P_{v}\right\rceil P_{v}$, then we do the same for $k \geq\left\lceil Q_{d, 1}^{*} / P_{v}\right\rceil$, and, hence, $Q_{d, 1}^{*} \leq Q_{c, 1}^{*} \leq \bar{Q}_{c}^{*}$.

COROLLARY 1: If $(c-p) P_{v} \geq R_{v}$, the only possible value of $Q_{c, 1}^{*}$ that is less than $Q_{d, 1}^{*}$ is $\left(\left\lceil Q_{d, 1}^{*} / P_{v}\right\rceil-1\right) P_{v}$.

PROOF: The proof follows from Expression (11) and Theorem 1. 
PROPOSITION 3: When $(c-p) P_{v}>R_{v}$ and $Q_{d, 1}^{*} \geq$ $\left(\left\lceil Q_{d, 1}^{*} / P_{v}\right\rceil-1\right) P_{v}+R_{v} /(c-p)$, then $Q_{c, 1}^{*} \geq Q_{d, 1}^{*}$.

PROOF: See Appendix A.4 for the proof.

Recall that $R_{v} /(c-p)$ is the quantity that justifies the cost of an additional truck for the vendor. Therefore, Proposition 3 suggests that if utilizing the last truck to carry $Q_{d, 1}^{*}$ units is justifiable, then $Q_{c, 1}^{*} \geq Q_{d, 1}^{*}$. Otherwise, as stated in Theorem 1, we have $Q_{c, 1}^{*} \geq\left(\left\lceil Q_{d, 1}^{*} / P_{v}\right\rceil-1\right) P_{v}$. In this case $Q_{c, 1}^{*}<Q_{d, 1}^{*}$ is possible and this occurs only if $Q_{c, 1}^{*}=\left(\left\lceil Q_{d, 1}^{*} / P_{v}\right\rceil-1\right) P_{v}$.

PROPOSITION 4: When $(c-p) P_{v}=R_{v}$ :

- If $Q_{d, 1}^{*} \neq\left\lceil Q_{d, 1}^{*} / P_{v}\right\rceil P_{v}$, then $\left\lceil Q_{d, 1}^{*} / P_{v}\right\rceil P_{v} \geq$ $Q_{c, 1}^{*} \geq\left(\left\lceil Q_{d, 1}^{*} / P_{v}\right\rceil-1\right) P_{v}$.

- If $Q_{d, 1}^{*}=\left\lceil Q_{d, 1}^{*} / P_{v}\right\rceil P_{v}$, then $Q_{c, 1}^{*}=Q_{d, 1}^{*}$.

PROOF: The proof is presented in Appendix A.4.

Proposition 4 states that when the revenue from a full truck load is just enough to cover the per truck cost, depending on whether $Q_{d, 1}^{*}$ is a full truck load or less than truck load, the value of $Q_{c, 1}^{*}$ is within the "neighborhood" of $Q_{d, 1}^{*}$. That is, if $Q_{d, 1}^{*}$ is not a full truck load, coordination can decrease it down to or increase it up to the closest full truck load. More interestingly, if $Q_{d, 1}^{*}$ is a full truck load, Proposition 4 implies that there is no need for coordination.

\subsubsection{Coordinated Solutions for Model I}

In this section, we propose two coordination mechanisms by which the buyer orders the centralized order quantity while achieving the expected profits from his/her decentralized solution. Propositions 6 and 7 describe the structure of the first coordination mechanism whereas Propositions 8 and 9 describe the structure of the second coordination mechanism.
Proposition 5 is useful in proving why such coordination mechanisms work. Propositions 7 and 9 identify the cases where the vendor can increase his/her profits by decreasing the buyer's order quantity. Hence, these propositions demonstrate that, unlike the earlier work on channel coordination, when transportation capacity considerations are modeled explicitly, the resulting cost function no longer exhibits economies of scale. In our following discussion, with a slight change of notation, we use $\bar{\Pi}_{b}(Q, c)$ for the expected buyer profit function. This is because the wholesale price $c$ will be specified by the vendor in such a way that ordering the centralized order quantity does not decrease the buyer's profits relative to his/her decentralized ordering policy. Therefore, we treat $c$ as a decision variable, and we let $\bar{Q}_{d}^{*}(\cdot)$ represent the optimal decentralized order quantity in Model I for a given value of the wholesale price. In the remainder of the text, as before, we let $\bar{Q}_{d}^{*}(c)=\bar{Q}_{d}^{*}$.

PROPOSITION 5: $\bar{\Pi}_{b}\left(\bar{Q}_{d}^{*}(c), c\right)$ is a decreasing function of $c$ for $c>v$.

PROOF: The proof is easy and is omitted.

\section{PROPOSITION 6: Let}

$\Delta_{1}=(r+b-v)\left[F\left(Q_{c, 1}^{*}\right)-F\left(\bar{Q}_{d}^{*}\right)\right] \quad$ and $\quad c_{1}=c-\Delta_{1}$.

If $Q_{c, 1}^{*}>\bar{Q}_{d}^{*}$, under a unit discount of $\Delta_{1}$ offered by the vendor to the buyer and a fixed payment of $\bar{\Pi}_{b}\left(Q_{c, 1}^{*}, c_{1}\right)-$ $\bar{\Pi}_{b}\left(\bar{Q}_{d}^{*}, c\right)$ made by the buyer to the vendor, the buyer stays in a no worse situation by ordering $Q_{c, 1}^{*}$ units.

PROOF: See Appendix A.5 for the proof.

We call the above coordination mechanism the two-part tariff schedule with fixed cost to the buyer. Figure 4 illustrates

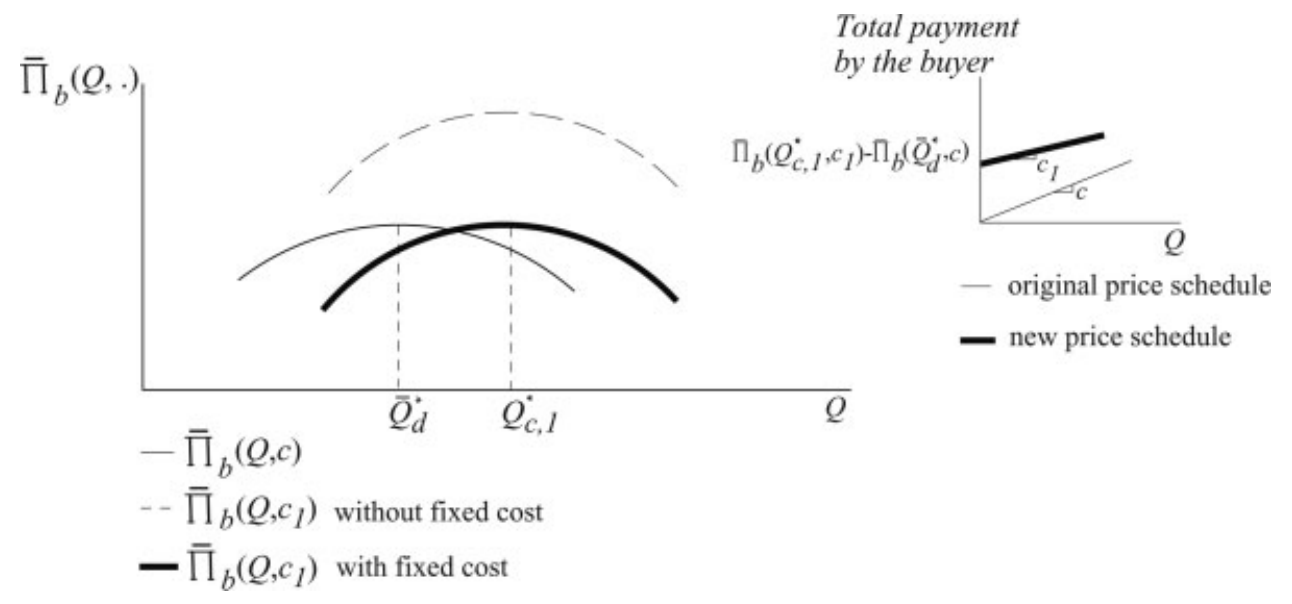

Figure 4. First coordination mechanism when $Q_{c, 1}^{*}>\bar{Q}_{d}^{*}$. 
the effects of the discounted price on the buyer's expected profits with, and without, the fixed payment. The dashed curve represents $\bar{\Pi}_{b}(Q)$ under the discounted price. As seen from Fig. 4, the maximizer of this curve is $Q_{c, 1}^{*}$. Therefore, the discount encourages the buyer to order the centralized quantity. However, as formally stated in Proposition 5, the buyer's expected profit under the discounted price is more than that in the decentralized solution under the original price. Therefore, the profit maximizing vendor, who wants to keep the buyer in an "indifferent" situation, charges him/her a fixed payment that results in the dark curve in Fig. 4. Note that this kind of schedule exhibits a decreasing marginal price.

\section{PROPOSITION 7: Let}

$$
\begin{aligned}
& \Delta_{2}=(r+b-v)\left[F\left(\bar{Q}_{d}^{*}\right)-F\left(Q_{c, 1}^{*}\right)\right], c_{2}=c+\Delta_{2}, \\
& \text { and } \alpha_{1}=\inf \{x: f(x)>0\} .
\end{aligned}
$$

If $Q_{c, 1}^{*}<\bar{Q}_{d}^{*}$ and $Q_{c, 1}^{*}>\alpha_{1}$, under a unit price increase of $\Delta_{2}$ and a fixed payment of $\bar{\Pi}_{b}\left(\bar{Q}_{d}^{*}, c\right)-\bar{\Pi}_{b}\left(Q_{c, 1}^{*}, c_{2}\right)$ made by the vendor to the buyer, the buyer stays in a no worse situation by ordering $Q_{c, 1}^{*}$ units.

PROOF: See Appendix A.5 for the proof.

Since the buyer is rewarded for his/her increased expenses, we call the coordination mechanism, stated in Proposition 7 and illustrated in Fig. 5, the two-part tariff schedule with fixed reward to the buyer. Note that this schedule exhibits an increasing marginal price.

The pricing schedules given in Propositions 6 and 7 coordinate the system in such a way that when the buyer orders the centralized order quantity, his/her expected profits are no less than he/she would otherwise earn. Note that both of these mechanisms require a change in unit price accompanied by a transfer of fixed payments between the parties. However, the transfer of fixed payments may be impractical in some settings. For this reason, next we propose two coordination mechanisms under which different unit prices are charged for different order quantities whereas no fixed payments are required.

PROPOSITION 8: Let

$$
\Delta_{3}=\frac{\bar{\Pi}_{b}\left(\bar{Q}_{d}^{*}, c\right)-\bar{\Pi}_{b}\left(Q_{c, 1}^{*}, c\right)}{Q_{c, 1}^{*}} \quad \text { and } \quad c_{3}=c-\Delta_{3} .
$$

If $Q_{c, 1}^{*}>\bar{Q}_{d}^{*}$, under a unit discount of $\Delta_{3}$ for order sizes greater than or equal to $Q_{c, 1}^{*}, Q_{c, 1}^{*}$ maximizes the buyer's expected profit function. Furthermore, $\bar{\Pi}_{b}\left(Q_{c, 1}^{*}, c_{3}\right)=$ $\bar{\Pi}_{b}\left(\bar{Q}_{d}^{*}, c\right)$.

PROOF: See Appendix A.5 for the proof.

The above coordination mechanism changes the price only after $Q_{c, 1}^{*}$. Therefore, the expected profit of the buyer at $\bar{Q}_{d}^{*}$ stays the same. This implies that the buyer is indifferent to a choice between $Q_{c, 1}^{*}$ and $\bar{Q}_{d}^{*}$. However, by slightly increasing the price for order sizes less than $Q_{c, 1}^{*}$, the vendor can change the behavior of the buyer so that the buyer orders $Q_{c, 1}^{*}$ units. The dashed curve in Fig. 6 shows how $\bar{\Pi}_{b}(Q)$ would appear under the discounted price without any price breaks. However, as seen in Fig. 6, in this case, the buyer's expected profits would be maximized at a quantity between $\bar{Q}_{d}^{*}$ and $Q_{c, 1}^{*}$. The price breakpoint that the vendor offers, however, encourages the buyer not to order this quantity. The dark continuous line in Fig. 6 shows the buyer's expected profits after a slightly increased unit price before $Q_{c, 1}^{*}$ and a discount after $Q_{c, 1}^{*}$. Since the discount is valid on all items for order sizes greater than or equal to $Q_{c, 1}^{*}$, we call this pricing schedule all-unit quantity pricing with economies of scale.

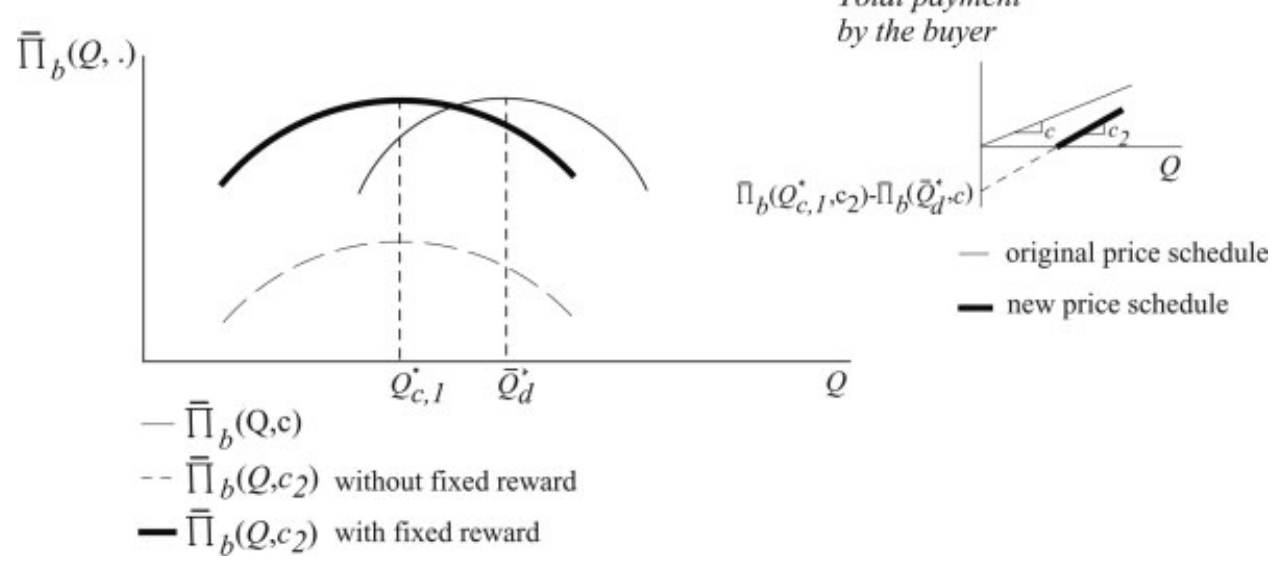

Figure 5. First coordination mechanism when $Q_{c, 1}^{*}<\bar{Q}_{d}^{*}$. 


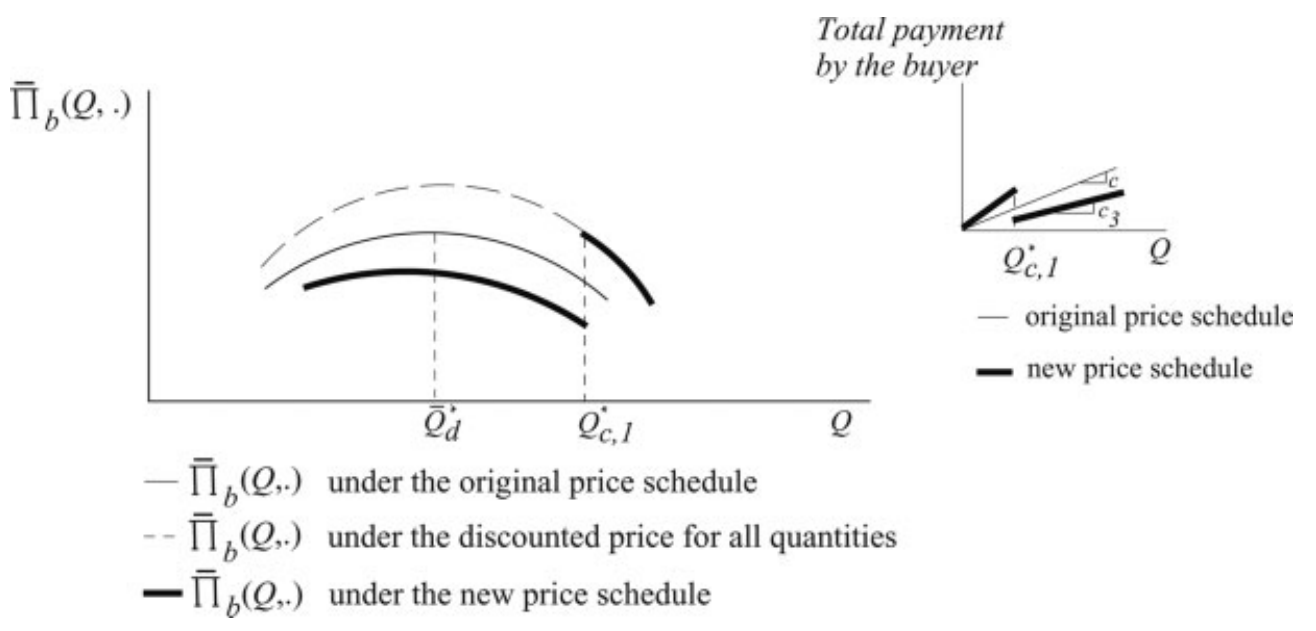

Figure 6. Second coordination mechanism when $Q_{c, 1}^{*}>\bar{Q}_{d}^{*}$.

PROPOSITION 9: Let

$$
\Delta_{4}=\frac{\bar{\Pi}_{b}\left(\bar{Q}_{d}^{*}, c\right)-\bar{\Pi}_{b}\left(Q_{c, 1}^{*}, c\right)}{Q_{c, 1}^{*}} \quad \text { and } \quad c_{4}=c-\Delta_{4} .
$$

If $Q_{c, 1}^{*}<\bar{Q}_{d}^{*}$, under a unit discount of $\Delta_{4}$ for order sizes less than $Q_{c, 1}^{*}, Q_{c, 1}^{*}$ maximizes the buyer's expected profit function. Furthermore, $\bar{\Pi}_{b}\left(Q_{c, 1}^{*}, c_{4}\right)=\bar{\Pi}_{b}\left(\bar{Q}_{d}^{*}, c\right)$.

PROOF: See Appendix A.5 for the proof.

With this coordination mechanism, the buyer is again indifferent to a choice between $Q_{c, 1}^{*}$ and $\bar{Q}_{d}^{*}$. However, by slightly increasing the unit price for order sizes greater than $Q_{c, 1}^{*}$, the vendor can again influence the behavior of the buyer so that he/she orders $Q_{c, 1}^{*}$ units (see Fig. 7). We call this coordination mechanism all-unit quantity pricing with diseconomies of scale.

We note that the coordination mechanisms proposed above can also be used for a system without truck capacities and costs. Recall that the only difference between such a system and the one considered in Model I is the consideration of transportation costs and capacities in the vendor's replenishment. As implied by Proposition 1 and stated in Remark 1, without a generalized replenishment cost structure for the vendor, the optimal order quantity in the centralized solution is always greater than, or equal to, the optimal order quantity in the decentralized solution. Therefore, to coordinate the system without truck capacities and costs, we do not need to consider the case where $Q_{c, 1}^{*}<\bar{Q}_{d}^{*}$. Since the buyer's cost structure is the same in both systems (i.e., Model I with, and without, transportation considerations), Proposition 6

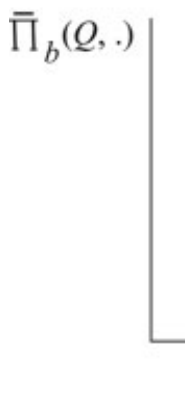

Total payment

by the buyer

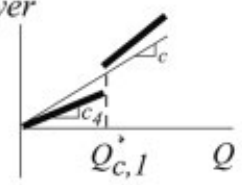

- original price schedule

- new price schedule

- $\bar{\Pi}_{b}(Q,$.$) under the original price schedule$

- - $\bar{\Pi}_{b}(Q,$.$) under the discounted price for all quantities$

- $\bar{\Pi}_{b}(Q,$.$) under the new price schedule$

Figure 7. Second coordination mechanism when $Q_{c, 1}^{*}<\bar{Q}_{d}^{*}$. 
and Proposition 8 can still be used by replacing $Q_{c, 1}^{*}$ with $\bar{Q}_{c}^{*}$.

\subsection{Model II}

\subsubsection{Decentralized Solution for Model II}

In the second model, we consider the generalized replenishment cost structure for the buyer as well. The buyer's subproblem is to maximize $\Pi_{b}(Q)=\bar{\Pi}_{b}(Q)-\left\lceil Q / P_{b}\right\rceil R_{b}$ over all $Q \geq 0$. As described in Section $4, \bar{\Pi}_{b}(Q)$ is a strictly concave function of $Q$ with a maximizer at $\bar{Q}_{d}^{*}$. Therefore, Proposition (2) can again be used for computing the maximizer of $\Pi_{b}(Q)$ by taking $g(Q)=\bar{\Pi}_{b}(Q)$ and $q=\bar{Q}_{d}^{*}$. Hence,

$$
Q_{d, 2}^{*}= \begin{cases}i P_{b} & \text { if } \mathcal{F} \neq \emptyset, \\ \bar{Q}_{d}^{*} & \text { if } \mathcal{F}=\emptyset \text { and } \Pi_{b}\left(\bar{Q}_{d}^{*}\right) \\ & >\Pi_{b}\left(\left(\left\lceil\frac{\bar{Q}_{d}^{*}}{P_{b}}\right\rceil-1\right) P_{b}\right), \\ \left(\left\lceil\frac{\bar{Q}_{d}^{*}}{P_{b}}\right\rceil-1\right) P_{b} & \text { otherwise, }\end{cases}
$$

where $\mathcal{F}=\left\{k \in Z^{+}:-\bar{\Pi}_{b}\left(k P_{b}\right)+\bar{\Pi}_{b}\left((k+1) P_{b}\right) \leq\right.$ $\left.R_{b},(k+1) P_{b} \leq \bar{Q}_{d}^{*}\right\}$ and $i=\min \{k$ s.t. $k \in \mathcal{F}\}$.

\subsubsection{Centralized Solution for Model II}

In the centralized solution, we maximize $\Pi_{c}^{I I}(Q)=$ $\Pi_{v}(Q)+\Pi_{b}(Q)$. Note that $\Pi_{v}(Q)=\bar{\Pi}_{v}(Q)-\left\lceil Q / P_{v}\right\rceil R_{v}$ and $\Pi_{b}(Q)=\bar{\Pi}_{b}(Q)-\left\lceil Q / P_{b}\right\rceil R_{b}$. Therefore, $\Pi_{c}^{I I}(Q)$ can be rewritten as

$$
\Pi_{c}^{I I}(Q)=\bar{\Pi}_{v}(Q)+\bar{\Pi}_{b}(Q)-\left\lceil\frac{Q}{P_{v}}\right\rceil R_{v}-\left\lceil\frac{Q}{P_{b}}\right\rceil R_{b}
$$

Note also that $\bar{\Pi}_{v}(Q)+\bar{\Pi}_{b}(Q)=\bar{\Pi}_{c}(Q)$. This leads to

$$
\Pi_{c}^{I I}(Q)=\bar{\Pi}_{c}(Q)-\left\lceil\frac{Q}{P_{v}}\right\rceil R_{v}-\left\lceil\frac{Q}{P_{b}}\right\rceil R_{b} .
$$

Recall that $\bar{\Pi}_{c}(Q)$ is the expected system profits of the centralized solution when no truck costs or capacity requirements are included. It is a concave function of $Q$ with a maximizer at $\bar{Q}_{c}^{*}$.

Based on the following properties of $\Pi_{c}^{I I}(Q)$, whose proofs are presented in Appendix A.6, we provide a finite time exact solution procedure for its maximization.

PROPERTY 10: Let $Q_{2}>Q_{1}>\bar{Q}_{c}^{*}$. Then $\Pi_{c}^{I I}\left(Q_{2}\right)<$ $\Pi_{c}^{I I}\left(Q_{1}\right)$. That is, $\Pi_{c}^{I I}(Q)$ is decreasing after $\bar{Q}_{c}^{*}$.

PROPERTY 11: Let $Q_{1}$ and $Q_{2}$ be such that $\left(k_{1}-1\right) P_{b}<$ $Q_{1}<Q_{2} \leq k_{1} P_{b} \leq \bar{Q}_{c}^{*}$ and $\left(k_{2}-1\right) P_{v}<Q_{1}<Q_{2} \leq$ $k_{2} P_{v} \leq \bar{Q}_{c}^{*}$ where $k_{1} \in Z^{+}$and $k_{2} \in Z^{+}$.Then $\Pi_{c}^{I I}\left(Q_{1}\right)<$
$\Pi_{c}^{I I}\left(Q_{2}\right)$. In other words, for $Q \leq \bar{Q}_{c}^{*}, \Pi_{c}^{I I}(Q)$ is piecewise increasing.

Therefore, in maximizing $\Pi_{c}^{I I}(Q)$, we must consider $\bar{Q}_{c}^{*}$ and the integer multiples of $P_{b}$ and $P_{v}$ that are less than or equal to $\bar{Q}_{c}^{*}$. Knowing how to obtain the centralized solution, next we discuss alternative coordination mechanisms for Model II.

\subsubsection{Coordinated Solutions for Model II/ Vendor-Managed Incentive and Delivery Contracts}

In this section, we propose two coordination mechanisms by which the buyer orders the centralized order quantity while achieving the expected profits from his/her decentralized solution. Propositions 10 and 11 describe the structure of the first coordination mechanism, which proposes the vendor to provide fixed payments/rewards to the buyer only for order sizes at specified intervals. The vendor can pass these incentives to the buyer by direct payments. Another alternative for the vendor is to pay some or all of the buyer's transportation costs using a VMD agreement as an incentive. Hence, the second coordination mechanism we propose for Model II relies on the idea of free deliveries to the buyer where the vendor pays for truck costs. Propositions 12 and 13 describe the structure of this mechanism.

PROPOSITION 10: If $Q_{c, 2}^{*}>Q_{d, 2}^{*}$, the following coordination mechanism maximizes the buyer's expected profit function with a maximum function value of $\Pi_{b}\left(Q_{d, 2}^{*}\right)$ at $Q_{c, 2}^{*}$.

- If $Q_{c, 2}^{*}>\bar{Q}_{d}^{*}$, the vendor pays the buyer a fixed fee of $\Pi_{b}\left(Q_{d, 2}^{*}\right)-\Pi_{b}\left(Q_{c, 2}^{*}\right)$ for orders larger than or equal to $Q_{c, 2}^{*}$.

- If $Q_{c, 2}^{*}<\bar{Q}_{d}^{*}$, the vendor pays the buyer a fixed fee of $\Pi_{b}\left(Q_{d, 2}^{*}\right)-\Pi_{b}\left(Q_{c, 2}^{*}\right)$ for order sizes in the range $\left(\left(\left\lceil Q_{c, 2}^{*} / P_{b}\right\rceil-1\right) P_{b}, Q_{c, 2}^{*}\right]$.

PROOF: See Appendix A.7 for the proof.

PROPOSITION 11: If $Q_{c, 2}^{*}<Q_{d, 2}^{*}$, the following coordination mechanism maximizes the buyer's expected profit function with a maximum function value of $\Pi_{b}\left(Q_{d, 2}^{*}\right)$ at $Q_{c, 2}^{*}$.

- If $Q_{c, 2}^{*}=k P_{b}$ for some positive integer $k$, then the vendor pays the buyer a fixed fee of $\Pi_{b}\left(Q_{d, 2}^{*}\right)-$ $\Pi_{b}\left(Q_{c, 2}^{*}\right)$ for order sizes less than or equal to $Q_{c, 2}^{*}$.

- If $Q_{c, 2}^{*}=k P_{v}$, the vendor pays the buyer a fixed fee of $\Pi_{b}\left(Q_{d, 2}^{*}\right)-\Pi_{b}\left(Q_{c, 2}^{*}\right)$ for order sizes in the range $\left(\left(\left\lceil Q_{c, 2}^{*} / P_{b}\right\rceil-1\right) P_{b}, Q_{c, 2}^{*}\right]$. 
PROOF: See Appendix A.7 for the proof.

Under the coordination mechanism described in Propositions 10 and 11, the vendor pays fixed rewards to the buyer, and, thus, it is called the vendor-managed incentive contract with fixed rewards to the buyer. This contract type can be viewed as a motivation for recent practices, known as vendormanaged inventory, where the vendor pays some or all of the transportation related expenses for the buyer. That is, the vendor can pass a reward for the buyer in alternative forms, such as by covering the buyer's transportation expenses.

The benefits of vendor-managed inventory have been discussed widely in the current literature and they extend beyond cost savings associated with transportation, e.g., see [6, 7,9-11,19,25,28]. However, to the best of our knowledge, the existing analytical work in the area does not provide a method for quantifying the potential gains through a carefully designed VMD contract under explicit truck costs. For this reason, next, we analyze a VMD contract for Model II, where the vendor agrees to pay for truck costs of the buyer. This alternative coordination mechanism is called the VMD contract with free shipping for the buyer, and it may result in extra savings for two reasons: (i) the per truck cost incurred/negotiated by the vendor may be less than that of the buyer, and/or (ii) the vendor may own a fleet of vehicles with larger truck capacity than that of the buyer. As before, our analysis for this type of contract takes the vendor's point of view. We assume that the terms of the contract is specified in a way that the vendor's expected profits are increased while the buyer stays in a no worse situation, i.e., the buyer's expected profits stays at $\Pi_{b}\left(Q_{d, 2}^{*}\right)$. On the other hand, when the vendor undertakes the transportation responsibility, the buyer may benefit from potential savings in his/her own fixed costs, i.e., $K_{b}$, due to a reduction in order processing expenses, etc. Hence, a carefully designed VMD contract may translate into real savings not only for the vendor but also for the buyer. For this reason, in some problem instances, the proposed VMD contract with free shipping may be preferable over the incentive contract described by Propositions 10 and 11 above.

Let $\Pi_{c}^{V M D}(Q)$ and $Q_{c, V M D}^{*}$ denote the expected system profit function under the proposed VMD contract and its maximizer, respectively. Then, we can write

$$
\Pi_{c}^{V M D}(Q)=\bar{\Pi}_{c}(Q)-\left\lceil\frac{Q}{P_{v}}\right\rceil R_{v}-\left\lceil\frac{Q}{P_{b}^{V M D}}\right\rceil R_{b}^{V M D},
$$

where $\bar{\Pi}_{c}(Q)$ is given by Expression (4), and $P_{b}^{V M D}$ and $R_{b}^{V M D}$ denote the truck capacity and per truck cost associated with the buyer's replenishment under the proposed VMD contract. In other words, the last term of Expression (15) represents the outbound truck costs paid by the vendor. Obviously, if the vendor utilizes the same kind of truck for both the inbound and the outbound transportation, then $P_{b}^{V M D}=P_{v}$. However, for the sake of generality, we do not restrict ourselves to this case.

Next, we argue that as long as the expected relative system gain is positive, i.e., $\Pi_{c}^{V M D}\left(Q_{c, V M D}^{*}\right)-\Pi_{c}^{I I}\left(Q_{c, 2}^{*}\right) \geq 0$, there is opportunity for additional savings under the proposed arrangement, and we present two propositions that specify the terms of the proposed VMD contract.

PROPOSITION 12: Let $\Delta_{5}=(r+b-v)\left[F\left(Q_{c, V M D}^{*}\right)-\right.$ $\left.F\left(\bar{Q}_{d}^{*}\right)\right]$ and $c_{5}=c-\Delta_{5}$. If $Q_{c, V M D}^{*} \geq \bar{Q}_{d}^{*}$, under a unit discount of $\Delta_{5}$ offered by the vendor to the buyer and a fixed payment of $\bar{\Pi}_{b}\left(Q_{c, V M D}^{*}, c_{5}\right)-\Pi_{b}\left(Q_{d, 2}^{*}, c\right)$ made by the buyer to the vendor, the buyer is expected to earn no less than $\Pi_{b}\left(Q_{d, 2}^{*}, c\right)$ by ordering $Q_{c, V M D}^{*}$ units.

PROOF: See Appendix A.7 for the proof.

PROPOSITION 13: Let $\Delta_{6}=(r+b-v)\left[F\left(\bar{Q}_{d}^{*}\right)-\right.$ $\left.F\left(Q_{c, V M D}^{*}\right)\right], c_{6}=c+\Delta_{6}$, and $\alpha_{1}=\inf \{x: f(x)>0\}$.

- If $Q_{c, V M D}^{*}<\bar{Q}_{d}^{*}, Q_{c, V M D}^{*}>\alpha_{1}$, and $\bar{\Pi}_{b}\left(Q_{c, V M D}^{*}\right.$, $\left.c_{6}\right)>\Pi_{b}\left(Q_{d, 2}^{*}, c\right)$, then under a unit price increase of $\Delta_{6}$ and a fixed payment of $\bar{\Pi}_{b}\left(Q_{c, V M D}^{*}, c_{6}\right)-$ $\Pi_{b}\left(Q_{d, 2}^{*}, c\right)$ made by the buyer to the vendor, the buyer is expected to earn no less than $\Pi_{b}\left(Q_{d, 2}^{*}, c\right)$ by ordering $Q_{c, V M D}^{*}$ units.

- If $Q_{c, V M D}^{*}<\bar{Q}_{d}^{*}, Q_{c, V M D}^{*}>\alpha_{1}$, and $\bar{\Pi}_{b}\left(Q_{c, V M D}^{*}\right.$, $\left.c_{6}\right)<\Pi_{b}\left(Q_{d, 2}^{*}, c\right)$, then under a unit price increase of $\Delta_{6}$ and a fixed payment of $\Pi_{b}\left(Q_{d, 2}^{*}, c\right)-$ $\bar{\Pi}_{b}\left(Q_{c, V M D}^{*}, c_{6}\right)$ made by the vendor to the buyer, the buyer is expected to earn no less than $\Pi_{b}\left(Q_{d, 2}^{*}, c\right)$ by ordering $Q_{c, V M D}^{*}$ units.

PROOF: See Appendix A.7 for the proof.

For the purpose of illustrating the potential gains under the proposed VMD arrangement, let us consider the case where $p=13, c=14, r=32, v=11, b=14, P_{b}=$ $200, R_{b}=250, P_{v}=250, R_{v}=300, K_{b}=350, K_{v}=400$, and the demand is exponentially distributed with parameter $\lambda=0.002$. It can be easily shown that the decentralized and centralized order quantities without the VMD contract, i.e., $Q_{d, 2}^{*}$ and $Q_{c, 2}^{*}$ values, respectively, are both given by 1000 units. Although this result suggests that the system is already coordinated, the buyer's and vendor's resulting expected profits are $\$ 3531.63$ and $-\$ 600$, respectively, i.e., the vendor's expected loss is $\$ 600$. Now, suppose that the vendor incurs $\$ 140$ per truck if he/she undertakes the buyer's truck costs (i.e., $R_{b}^{V M D}=140$ ). Then, Proposition 13 suggests that under a unit price increase of 1.737 and a fixed payment of $\$ 486.74$ from the vendor to the buyer, the buyer's expected profits remain at $\$ 3531.63$ whereas the vendor's expected 
profits increase to $\$ 90$. Furthermore, for this specific example, the VMD contract does not even require the buyer to change his/her order quantity, i.e., $Q_{c, V M D}^{*}=1000$. As this example suggests, under the proposed VMD arrangement, the vendor can achieve a substantial gain by turning a nonprofitable business into a profitable one whereas the buyer remains in a no worse situation in terms of the expected profits. However, when the vendor undertakes the buyer's transportation operations, this may lead to a reduction in the buyer's fixed cost $K_{b}$ so that his/her actual expected profit exceeds the benchmark level of $\$ 3531.63$. Hence, we say that the proposed VMD arrangement may lead to a "truly win-win solution" for both parties. In fact, this is the fundamental characteristic that distinguishes the proposed VMD contract from the other coordination mechanisms discussed in this paper as well as in the previous literature.

For the example discussed above, Fig. 8 illustrates the expected relative system gain, as a function of $R_{b}^{V M D}$. Observe that both the expected relative gain and its rate are decreasing in $R_{b}^{V M D}$. Figure 8 demonstrates two threshold points for $R_{b}^{V M D}$, each defining a region over which the corresponding $Q_{c, V M D}^{*}$ values and the rate of expected relative gain remain constant. It is worthwhile to note that, for the particular example we consider, the expected relative system gain is positive not only for $R_{b}^{V M D}=140<R_{b}=250$ but for all $R_{b}^{V M D} \leq 312.5$. Obviously, the expected system gain also depends on the value of $P_{b}^{V M D}$ relative to $P_{b}$. Hence, we conclude this section by analyzing the cases under which the expected relative system gain proves to be profitable.

\section{PROPOSITION 14:}

- Case 1. If $P_{b}^{V M D} \geq P_{b}$, and $R_{b}^{V M D} \leq R_{b}$, then $\Pi_{c}^{V M D}\left(Q_{c, V M D}^{*}\right) \geq \Pi_{c}^{I I}\left(Q_{c, 2}^{*}\right)$.

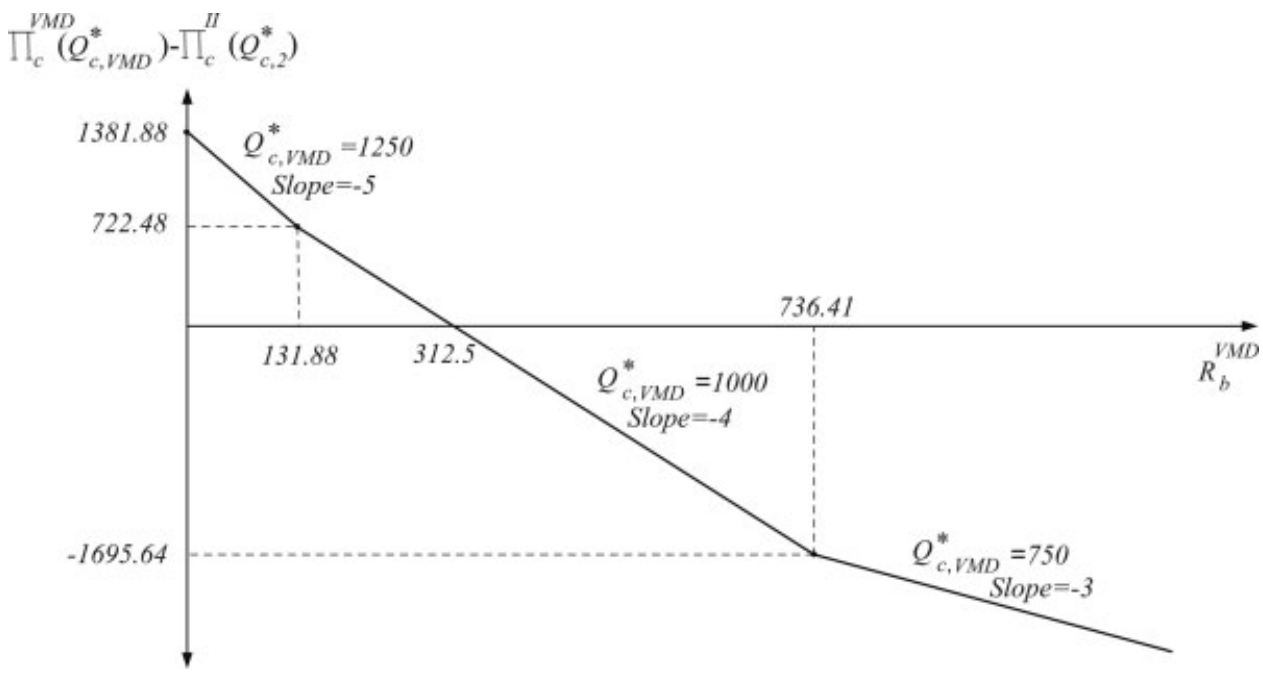

Figure 8. An illustration of relative gain under the proposed VMD contract.
- Case 2. If $P_{b}^{V M D} \leq P_{b}$, and $R_{b}^{V M D} \geq R_{b}$, then $\Pi_{c}^{V M D}\left(Q_{c, V M D}^{*}\right) \leq \bar{\Pi}_{c}^{I I}\left(Q_{c, 2}^{*}\right)$.

- In other cases, i.e., if

Case 3. $P_{b}^{V M D} \geq P_{b}$, and $R_{b}^{V M D}>R_{b}$, or

Case 4. $P_{b}^{V M D} \leq P_{b}$, and $R_{b}^{V M D}<R_{b}$, we either have $\Pi_{c}^{V M D}\left(Q_{c, V M D}^{*}\right)>\Pi_{c}^{I I}\left(Q_{c, 2}^{*}\right)$ or $\Pi_{c}^{V M D}\left(Q_{c, V M D}^{*}\right)<\Pi_{c}^{I I}\left(Q_{c, 2}^{*}\right)$.

PROOF: The proof is presented in Appendix A.7.

\section{SUMMARY OF INSIGHTS AND FUTURE RESEARCH DIRECTIONS}

Channel coordination is particularly important in competitive end-customer markets where the prices are close to the product's marginal cost and the market sets the price. Therefore, reducing other costs, such as logistics-related expenses, is important for the vendor to increase his/her profits. In fact, transportation costs represent a significant portion of the logistics-related expenses, and, consequently, substantial savings can be achieved by the vendor by incorporating transportation considerations with the channel coordination objective.

For the problem of interest in this paper, the vendor's expected profit is no longer an increasing function of the buyer's order quantity. Therefore, the premise that provided a foundation for the earlier work (i.e., Proposition 1) does not hold. Consequently, new coordination mechanisms must be designed to induce the buyer to decrease his/her order quantity in some cases. To this end, the paper proposes different variants of wholesale pricing and fixed payments as means to achieve coordinated solutions while answering four important questions, namely questions Q1, Q2, Q3, and Q4 listed in Section 1, as we summarize below. 
In Model I, only the vendor is subject to the generalized replenishment cost given by Expression (1). Some of the proposed pricing schemes for this model (i.e., "two-part tariff schedule with fixed cost to the buyer" and "all-unit quantity pricing with economies of scale") coordinate the supply chain by increasing the buyer's order quantity while the others (i.e., "all-unit quantity pricing with diseconomies of scale" and "two-part tariff schedule with fixed reward to the buyer") coordinate the channel by decreasing the buyer's order quantity. In Model II, both the vendor and the buyer are subject to the generalized replenishment cost given by Expression (1). In this case, the buyer's expected profit function is no longer continuous. Due to the complex piecewise structure of the buyer's expected profit function, we propose that the vendor makes fixed payments to the buyer only for order sizes at specified intervals. The vendor can pass these incentives to the buyer either by direct payments/incentives or by agreeing to pay the buyer's transportation costs as in the case of a VMD agreement. Hence, we also analyze a specific VMD contract where the vendor agrees to send free deliveries by covering the buyer's truck costs. We quantify the value of such a contract and demonstrate that it may provide a substantial gain for the buyer-vendor system. We argue that the proposed VMD contract may lead to truly win-win solutions, and we provide numerical and analytical results justifying the benefits of VMD practices.

Furthermore, using the coordination mechanisms proposed, we identify additional opportunities for the vendor to improve his/her profits by potentially turning a nonprofitable transaction into a profitable one. If the revenue associated with one full truck load does not exceed the truck cost (i.e., $\left.(c-p) P_{v} \leq R_{v}\right)$, the vendor is at loss. However, he/she can still decrease the magnitude of these losses through coordination. If the marginal value of one full truck load of items is more than the truck cost (i.e., $(c-p) P_{v}>R_{v}$ ), we show that the vendor has the opportunity even to profit from a transaction that previously resulted in a loss (see Properties 5, 6, and 7).

In fact, the relative values of $(c-p) P_{v}$ and $R_{v}$ also provide important results for a third party observing the buyer-vendor system in Model I. Knowing the relationship between $(c-$ p) $P_{v}$ and $R_{v}$, in addition to the buyer's decentralized order quantity, Theorem 1, Proposition 3, and Proposition 4 provide upper and lower bounds for the order quantity in the coordinated solution. A third party transportation provider can use these results to influence the coordinated solution by changing the parameter values under his/her control, i.e., $P_{v}$ and $R_{v}$.

Another important result of our analysis is stated in Property 8 and Remark 2, which compare the coordinated solutions of Model I with, and without, consideration of transportation capacity and costs. This result implies that the general cost structure may, or may not, have an effect on the coordinated solution. If it has an effect, then coordination is achieved at a full truck load. A similar result also holds for Model II, where in the latter case, the coordinated solution is achieved either at an integer multiple of the vendor's truck size or an integer multiple of the buyer's truck size. This result is also useful for a third party transportation provider because, if a coordination mechanism is in place between a buyer and a vendor as proposed in this paper, the transportation provider should expect more FTL orders under a delivery price schedule as in Expression (1). Consequently, for the sake of truck utilization, it seems reasonable for the transportation provider to assess freight charges using a delivery price schedule as in Expression (1), rather than one of the other well-known freight schedules, such as an all-units or an incremental delivery-quantity discount schedule.

It is worthwhile to note that the analysis in this paper takes the vendor's viewpoint and assumes that the vendor has prior information about the buyer's reactions or that the parameters of the problem are common knowledge. Under this assumption, the paper demonstrates that there are significant cost saving opportunities in systems with explicit cargo costs and capacity. These opportunities extend beyond those associated with more traditional systems (e.g., the system considered in [26]). Important extensions of the problem considered here take the viewpoint of the buyer and incorporate information asymmetry considerations in the context of contract design for VMD. Other notable generalizations include game theoretic models of (i) other contractual settings such as revenue-sharing contract, (ii) cases of multiple buyers and/or multiple vendors, and (iii) buyer-vendor-transporter problems.

\section{APPENDIX A}

\section{A.1. Proof of Proposition 1}

By definition, we have $\pi_{b}^{c}=\Pi_{b}\left(Q_{c}^{*}\right), \pi_{v}^{c}=\Pi_{v}\left(Q_{c}^{*}\right), \pi_{b}^{d}=\Pi_{b}\left(Q_{d}^{*}\right)$, and $\pi_{v}^{d}=\Pi_{v}\left(Q_{d}^{*}\right)$. We know that $\pi^{d} \leq \pi^{c}$, and, therefore, $\pi_{b}^{d}+\pi_{v}^{d} \leq$ $\pi_{b}^{c}+\pi_{v}^{c}$. Since we also have $\pi_{b}^{d} \geq \pi_{b}^{c}$, we can write $\pi_{v}^{c}-\pi_{v}^{d} \geq 0$, and, thus, $\Pi_{v}\left(Q_{c}^{*}\right) \geq \Pi_{v}\left(Q_{d}^{*}\right)$. When $\Pi_{v}(Q)$ is an increasing function of $Q$, this result implies that $Q_{c}^{*} \geq Q_{d}^{*}$.

\section{A.2. Properties of $h(Q)$ in Expression (7)}

PROOF OF PROPERTY 1: By definition, $q$ is the smallest maximizer of $g(Q)$. Therefore, $\forall Q>l P$ we have $g(Q) \leq g(q)$. Note that $\forall Q>l P$, we also have $\lceil Q / P\rceil>l$. It follows that

$$
g(Q)-\left\lceil\frac{Q}{P}\right\rceil R<g(q)-l P,
$$

so that $h(Q)<h(q)$.

PROOF OF PROPERTY 2: Since $g(Q)$ is a concave function, $\forall Q_{1}$, $Q_{2}$ s.t. $Q_{1}<Q_{2} \leq q$, we have $g\left(Q_{1}\right)<g\left(Q_{2}\right)$. That is, a concave function is increasing before its smallest maximizer. If $(k-1) P<Q_{1}<Q_{2} \leq k P$ then $\left\lceil Q_{1} / P\right\rceil=\left\lceil Q_{2} / P\right\rceil=k$. It follows that $g\left(Q_{1}\right)-\left\lceil Q_{1} / P\right\rceil R<$ $g\left(Q_{2}\right)-\left\lceil Q_{2} / P\right\rceil R$, and, hence, $h\left(Q_{1}\right)<h\left(Q_{2}\right)$. 
PROOF OF PROPERTY 3: Since $g(Q)$ is concave, it is decreasing (nonincreasing) after $q$. That is, $g\left(Q_{2}\right) \leq g\left(Q_{1}\right), \forall Q_{1}, Q_{2}$ s.t. $Q_{2}>Q_{1}>q$. It follows that $\left\lceil Q_{2} / P\right\rceil R \geq\left\lceil Q_{1} / P\right\rceil R$, and hence, $g\left(Q_{2}\right)-\left\lceil\frac{Q_{2}}{P}\right\rceil R \leq$ $g\left(Q_{1}\right)-\left\lceil\frac{Q_{1}}{P}\right\rceil R$. This implies that $h\left(Q_{2}\right) \leq h\left(Q_{1}\right)$.

PROOF OF PROPOSITION 2: We present the proof considering the following different cases.

- Case I: $\mathcal{F} \neq \varnothing$

Subcase I.I: $g((i+1) P)-g(i P)<R$ Since $g(Q)$ is a concave function, it follows that

$$
g((j+1) P)-g(j P)<R, \forall j \geq i \text { s.t. }(j+1) P \leq q,
$$

and

$$
g(q)-g((l-1) P)<R .
$$

Since $h((j+1) P)-h(j P)=g((j+1) P)-g(j P)-R$, Expression (16) implies that $h((j+1) P)-h(j P)<0$, $\forall j \geq i$ s.t. $(j+1) P \leq q$. That is, the value of $h(Q)$ at integer multiples of $P$ starts to decrease after $i P$. Therefore, $h(i P)>h((i+1) P), h(i P)>h((i+2) P), \ldots, h(i P)>$ $h((l-1) P)$. Recall that, $i$ is the smallest integer multiple of $P$ for which $g((i+1) P)-g(i P) \leq R$. This implies that $g((j+1) P)-g(j P)>R, \forall j<i$, and hence, $h(i P)>$ $h(j P), \forall j<i$.

At this point, we have eliminated all integer multiples of $P$ before $q$ except $i P$. Observe that, using Expression (17), $h(q)<h((l-1) P)$. Since we already have $h(i P)>h((l-$ 1) $P)$, it follows that $h(i P)>h(q)$. Hence, $Q^{*}=i P$.

Subcase I.II: $g((i+1) P)-g(i P)=R$

With the same reasoning as in the first subcase, we can eliminate all integer multiples of $P$ that are less than $i P$. For the remaining feasible points in set $\mathcal{A}^{3}$, let us first consider the integer multiples of $P$ between $i P$ and $q$. Let $m$ denote the largest integer $k$ such that $g((k+1) P)-g(k P)=R$ and $(k+1) P \leq q$ hold. Then, $\forall k$ such that $i \leq k \leq m$, we have $g((k+1) P)-g(k P)=R$ and $h(k P)=h((k+1) P)$. This implies that $h(i P)=h((i+1) P)=\ldots=h(m P)=$ $h((m+1) P)$. On the other hand, for all $j \geq(m+1)$ and $(j+1) P \leq q$, it follows from the concavity of $g(Q)$ that $g((j+1) P)-g(j P)<R$. The rest of the proof is similar to that of the previous subcase, and it relies on showing that (i) $h((m+1) P)>h(j P)$, for all integer $j$ such that $j>m+1$ and $j P \leq q$, and (ii) $h(q)<h((m+1) P)$. It follows that $i P,(i+1) P, \ldots, m P,(m+1) P$ are alternative solutions, and hence, $Q^{*}=i P$.

\section{- Case II: $\mathcal{F}=\emptyset$}

Since $\mathcal{F}=\emptyset$, then $g(k P)-g((k+1) P)>R, \forall k$ s.t. $(k+1) P \leq$ $q$. Therefore, $h((l-1) P)<h(j P), \forall j<(l-1)$. This implies that we can eliminate all integer multiples of $P$ that are less than $(l-1) P$ from set $\mathcal{A}^{3}$. Hence, either $(l-1) P$ or $q$ is optimum. If $h(q)>h((l-1) P)$, then $Q^{*}=q$. Else, if $h(q) \leq h((l-1) P)$, then $Q^{*}=(l-1) P$.

\section{A.3. Properties of $\Pi_{v}(Q)$ in Expression (6)}

PROOF OF PROPERTY 5: It follows from Property 4 that $\Pi_{v}\left(k P_{v}\right)>$ $\Pi_{v}(Q), \forall Q$ s.t. $(k-1) P_{v}<Q \leq k P_{v}$ where $k \in Z^{+}$. From Expression (6), we also have $\Pi_{v}\left(k P_{v}\right)=k\left[(c-p) P_{v}-R_{v}\right]-K_{v}$. If $(c-p) P_{v} \leq R_{v}$, then $\Pi_{v}\left(k P_{v}\right)<0, \forall k \in Z^{+}$. Since $\Pi_{v}\left(k P_{v}\right)>\Pi_{v}(Q), \forall Q$ s.t. $(k-1) P_{v}<$ $Q \leq k P_{v}$ and $k \in Z^{+}$, we also have $\Pi_{v}(Q)<0, \forall Q \geq 0$.

For the second part of this property, consider $Q=k P_{v}$ where $k=$ $\left\lceil K_{v} /\left((c-p) P_{v}-R_{v}\right)\right\rceil$. Note that $k$ in the above expression is the minimum number of fully loaded trucks used by the vendor so that he/she can compensate the fixed cost $K_{v}$ by the sales of all items purchased in this replenishment.

Since $(c-p) P_{v}>R_{v}$, we have $k \in Z^{+}$. Therefore,

$$
\begin{aligned}
\Pi_{v}\left(k P_{v}\right)=\left\lceil K_{v} /\left((c-p) P_{v}-R_{v}\right)\right\rceil\left[(c-p) P_{v}-R_{v}\right]-K_{v} \\
\quad \geq\left(K_{v} /\left((c-p) P_{v}-R_{v}\right)\right)\left[(c-p) P_{v}-R_{v}\right]-K_{v}=0 .
\end{aligned}
$$

Furthermore, $\Pi_{v}\left(k P_{v}\right)>0$ unless $K_{v}=k\left((c-p) P_{v}-R_{v}\right)$ in which case the vendor's profit is just neutral when selling $Q=k P_{v}$ units. However, by increasing his/her order quantity, say to $Q=(k+1) P_{v}$ units, the vendor can profit $(c-p) P_{v}-R_{v}$ dollars.

PROOF OF PROPERTY 6: From Expression (6), we have

$$
\begin{aligned}
\Pi_{v}\left((k+1) P_{v}\right)-\Pi_{v}\left(k P_{v}\right) & =(c-p)(k+1) P_{v}-K_{v}-(k+1) R_{v} \\
- & (c-p) k P_{v}+K_{v}+k R_{v}=(c-p) P_{v}-R_{v} .
\end{aligned}
$$

Since $(c-p) P_{v}>R_{v}$, it follows that $(c-p) P_{v}-R_{v}>0$ and, hence, $\Pi_{v}\left((k+1) P_{v}\right)-\Pi_{v}\left(k P_{v}\right)>0$.

PROOF OF PROPERTY 7: Since $Q<k P_{v}+R_{v} /(c-p)$, we have ( $c-$ p) $Q<(c-p) k P_{v}+R_{v}$. This, in turn, implies that $(c-p) Q-(k+1) R_{v}<$ $(c-p) k P_{v}-k R_{v}$, and subtracting $K_{v}$ from both sides of this inequality leads to

$$
(c-p) Q-K_{v}-(k+1) R_{v}<(c-p) k P_{v}-K_{v}-k R_{v} .
$$

By assumption, $k P_{v}<Q<k P_{v}+R_{v} /(c-p)$ and $(c-p) P_{v}>R_{v}$ (i.e., $\left.R_{v} /(c-p)<P_{v}\right)$, so that we also have $\left\lceil Q / P_{v}\right\rceil=k+1$. Therefore, $(c-p) Q-K_{v}-\left\lceil Q / P_{v}\right\rceil R_{v}<(c-p) k P_{v}-K_{v}-k R_{v}$, which implies that

$$
\Pi_{v}\left(k P_{v}\right)>\Pi_{v}(Q), \quad \forall Q \text { s.t. } k P_{v}<Q<k P_{v}+\frac{R_{v}}{c-p} .
$$

Note that if $Q=k P_{v}+R_{v} /(c-p)$, then

$$
\begin{aligned}
\Pi_{v}(Q)=(c-p) k P_{v}+R_{v}-K_{v} & -(k+1) R_{v} \\
& =(c-p) k P_{v}-K_{v}-k R_{v}=\Pi_{v}\left(k P_{v}\right) .
\end{aligned}
$$

\section{A.4. Centralized Solution for Model I: Proofs}

\section{PROOF OF PROPERTY 8:}

1. Expression (10) implies that $\Pi_{c}^{I}(Q)<\bar{\Pi}_{c}(Q) \forall Q>0$. As a result,

$$
\max _{Q>0} \Pi_{c}^{I}(Q)=\Pi_{c}^{I}\left(Q_{c, 1}^{*}\right)<\bar{\Pi}_{c}\left(Q_{c, 1}^{*}\right) \leq \max _{Q>0} \bar{\Pi}_{c}(Q)=\bar{\Pi}_{c}\left(\bar{Q}_{c}^{*}\right) .
$$

2. Again, using Eq. (10) we can write

$$
\begin{aligned}
\max _{Q>0} \Pi_{c}^{I}(Q)=\max _{Q>0}\left\{\bar{\Pi}_{c}(Q)-\right. & \left.\left\lceil\frac{Q}{P_{v}}\right\rceil R_{v}\right\} \\
& \leq \max _{Q>0} \bar{\Pi}_{c}(Q)-\min _{Q>0}\left\lceil\frac{Q}{P_{v}}\right\rceil R_{v} .
\end{aligned}
$$

Since $\min _{Q>0}\left\lceil Q / P_{v}\right\rceil R_{v}=R_{v}$, the above inequality leads to $\Pi_{c}^{I}\left(Q_{c, 1}^{*}\right) \leq \bar{\Pi}_{c}\left(\bar{Q}_{c}^{*}\right)-R_{v}$. Consequently, if $\bar{\Pi}_{c}\left(\bar{Q}_{c}^{*}\right)<R_{v}$, then $\Pi_{c}^{I}\left(Q_{c, 1}^{*}\right)<0$. 
PROOF OF PROPERTY 9: Recalling Expression (4), we can write

$$
\begin{aligned}
& -\bar{\Pi}_{c}\left(k P_{v}\right)+\bar{\Pi}_{c}\left((k+1) P_{v}\right)=(v-p) P_{v}+(r-v+b) \\
& \quad \times \int_{k P_{v}}^{(k+1) P_{v}}\left(x-k P_{v}\right) f(x) d x+(r-v+b) P_{v}\left[1-F\left((k+1) P_{v}\right)\right] .
\end{aligned}
$$

Also, if $k \in \mathcal{F}^{o}$, then $-\bar{\Pi}_{c}\left(k P_{v}\right)+\bar{\Pi}_{c}\left((k+1) P_{v}\right) \leq R_{v}$. Combining these two expressions and rearranging the terms leads to (12).

PROOF OF THEOREM 1: Using Expression (9) and the fact that $\bar{\Pi}_{v}(Q)=(c-p) Q-K_{v}$, we have

$$
\Pi_{c}^{I}(Q)=\bar{\Pi}_{b}(Q)+(c-p) Q-K_{v}-\left\lceil Q / P_{v}\right\rceil R_{v} .
$$

Recall that $\bar{\Pi}_{b}(Q)$ is a strictly concave function with a maximizer at $\bar{Q}_{d}^{*}$. Since truck capacity and costs are ignored for the buyer in Model I, $Q_{d, 1}^{*}=\bar{Q}_{d}^{*}$. Let $j=\left\lceil Q_{d, 1}^{*} / P_{v}\right\rceil$.

- For the first part of the proof, we will show that $\forall m \in Z^{+}$and $m<j-1, \Pi_{c}^{I}\left(m P_{v}\right)<\Pi_{c}^{I}\left((j-1) P_{v}\right)$. Let's consider $\Pi_{c}^{I}((j-$ 1) $P)-\Pi_{c}^{I}(m P)=\bar{\Pi}_{b}\left((j-1) P_{v}\right)-\bar{\Pi}_{b}\left(m P_{v}\right)+\left[(c-p) P_{v}-\right.$ $\left.R_{v}\right](j-1-m)$. Since $Q_{d, 1}^{*}$ is the maximizer of $\bar{\Pi}_{b}(Q)$ and $\bar{\Pi}_{b}(Q)$ is a strictly concave function of $Q, \bar{\Pi}_{b}\left((j-1) P_{v}\right)-\bar{\Pi}_{b}\left(m P_{v}\right)>0$. When $(c-p) P_{v}>R_{v}$, it is also true that $\left[(c-p) P_{v}-R_{v}\right](j-$ $1-m)>0$. Therefore, $\Pi_{c}^{I}\left((j-1) P_{v}\right)-\Pi_{c}^{I}\left(m P_{v}\right)>0$. Hence, $Q_{c, 1}^{*} \geq(j-1) P_{v}$

- For the second part of the proof, we will show that $\forall m \in Z^{+}$and $m<j, \Pi_{c}^{I}\left(m P_{v}\right)<\Pi_{c}^{I}\left(j P_{v}\right)$. For this purpose, let's consider the difference $\Pi_{c}^{I}\left(j P_{v}\right)-\Pi_{c}^{I}\left(m P_{v}\right)=\bar{\Pi}_{b}\left(j P_{v}\right)-\bar{\Pi}_{b}\left(m P_{v}\right)+[(c-$ p) $\left.P_{v}-R_{v}\right](j-m)$. Using the concavity of $\bar{\Pi}_{b}(Q)$ and the fact that $m P_{v}<j P_{v}=Q_{d, 1}^{*}$, we have that $\bar{\Pi}_{b}\left(j P_{v}\right)-\bar{\Pi}_{b}\left(m P_{v}\right)>0$. When $(c-p) P_{v}>R_{v},\left[(c-p) P_{v}-R_{v}\right](j-m)>0$. Therefore, $\Pi_{c}^{I}\left(j P_{v}\right)-\Pi_{c}^{I}\left(m P_{v}\right)>0$ and, hence, if $Q_{d, 1}^{*}=\left\lceil Q_{d}^{*} / P_{v}\right\rceil P_{v}$, then $Q_{c, 1}^{*} \geq Q_{d, 1}^{*}$.

PROOF OF PROPOSITION 3: As stated in Corollary 1, when $(c-$ p) $P_{v} \geq R_{v}$, the only possible value of $Q_{c, 1}^{*}$ that is less than $Q_{d, 1}^{*}$ is $\left(\left\lceil Q_{d, 1}^{*} / P_{v}\right\rceil-1\right) P_{v}$. Since $(c-p) P_{v}>R_{v}$, from Property 7 , we have

$$
\begin{aligned}
\Pi_{v}(Q) \geq \Pi_{v}\left(\left(\left\lceil\frac{Q_{d, 1}^{*}}{P_{v}}\right\rceil-1\right) P_{v}\right), \quad \forall Q \text { s.t. }\left\lceil Q_{d, 1}^{*} / P_{v}\right\rceil P_{v} \\
\geq Q>\left(\left\lceil Q_{d, 1}^{*} / P_{v}\right\rceil-1\right) P_{v}+R_{v} /(c-p) .
\end{aligned}
$$

Therefore, $\Pi_{v}\left(Q_{d, 1}^{*}\right) \geq \Pi_{v}\left(\left(\left\lceil Q_{d, 1}^{*} / P_{v}\right\rceil-1\right) P_{v}\right)$. Since $Q_{d, 1}^{*}$ is the maximizer of $\bar{\Pi}_{b}(Q)$, we also have

$$
\bar{\Pi}_{b}\left(Q_{d, 1}^{*}\right)>\bar{\Pi}_{b}\left(\left(\left\lceil\frac{Q_{d, 1}^{*}}{P_{v}}\right\rceil-1\right) P_{v}\right) .
$$

Hence, from Expression (8), we conclude that

$$
\Pi_{c}^{I}\left(Q_{d, 1}^{*}\right)>\Pi_{c}^{I}\left(\left(\left\lceil\frac{Q_{d, 1}^{*}}{P_{v}}\right\rceil-1\right) P_{v}\right) .
$$

\section{PROOF OF PROPOSITION 4:}

- As illustrated in Fig. 3a, if $(c-p) P_{v}=R_{v}$, then $\Pi_{v}\left(k P_{v}\right) \geq$ $\Pi_{v}(Q), \forall Q$ and $\forall k \in Z^{+}$. Therefore, $\Pi_{v}(Q)$ is maximized at both $Q=\left\lceil Q_{d, 1}^{*} / P_{v}\right\rceil P_{v}$ and $Q=\left(\left\lceil Q_{d, 1}^{*} / P_{v}\right\rceil-1\right) P_{v}$. Hence, $\forall Q>0$ we have

$$
\Pi_{v}\left(\left\lceil\frac{Q_{d, 1}^{*}}{P_{v}}\right\rceil P_{v}\right) \geq \Pi_{v}(Q),
$$

and

$$
\Pi_{v}\left(\left(\left\lceil\frac{Q_{d, 1}^{*}}{P_{v}}\right\rceil-1\right) P_{v}\right) \geq \Pi_{v}(Q)
$$

Let's first consider $Q>\left\lceil Q_{d, 1}^{*} / P_{v}\right\rceil P_{v}$. Since $\bar{\Pi}_{b}(Q)$ is a strictly concave function with a maximizer at $Q_{d, 1}^{*}$ and $Q_{d, 1}^{*} \leq\left\lceil Q_{d, 1}^{*} /\right.$ $\left.P_{v}\right\rceil P_{v}<Q$, we have

$$
\bar{\Pi}_{b}\left(\left\lceil\frac{Q_{d, 1}^{*}}{P_{v}}\right\rceil P_{v}\right)>\bar{\Pi}_{b}(Q) .
$$

Using Expressions (8), (19), and (21), we conclude that

$$
\Pi_{c}^{I}\left(\left\lceil\frac{Q_{d, 1}^{*}}{P_{v}}\right\rceil P_{v}\right)>\Pi_{c}^{I}(Q), \quad \forall Q>\left\lceil\frac{Q_{d, 1}^{*}}{P_{v}}\right\rceil P_{v} .
$$

With a similar argument, it can also be shown that

$$
\begin{aligned}
\Pi_{c}^{I}\left(\left(\left\lceil\frac{Q_{d, 1}^{*}}{P_{v}}\right\rceil-1\right) P_{v}\right) & >\Pi_{c}^{I}(Q), \\
\forall Q & <\left(\left\lceil\frac{Q_{d, 1}^{*}}{P_{v}}\right\rceil-1\right) P_{v} .
\end{aligned}
$$

Therefore, $\left\lceil Q_{d, 1}^{*} / P_{v}\right\rceil P_{v} \geq Q_{c, 1}^{*} \geq\left(\left\lceil Q_{d, 1}^{*} / P_{v}\right\rceil-1\right) P_{v}$.

- If $Q_{d, 1}^{*}=\left\lceil Q_{d, 1}^{*} / P_{v}\right\rceil P_{v}$, since both $\bar{\Pi}_{b}(Q)$ and $\Pi_{v}(Q)$ are maximized at $Q=Q_{d, 1}^{*}, \Pi_{c}^{I}(Q)$ is also maximized at this value. Therefore, $Q_{c, 1}^{*}=Q_{d, 1}^{*}$.

\section{A.5. Coordinated Solutions for Model I: Proofs}

PROOF OF PROPOSITION 6: By assumption, demand (i.e., $X$ ) is a continuous nonnegative random variable. We define $\alpha_{1}=\inf \{x: f(x)>0\}$ and $\alpha_{2}=\sup \{x: f(x)>0\}$. Therefore, $f(x)>0$ where $\alpha_{1}<x<\alpha_{2}$. Under the classical newsvendor assumptions, it is true that $\alpha_{1}<\bar{Q}_{d}^{*}<\alpha_{2}$ and $\alpha_{1}<\bar{Q}_{c}^{*}<\alpha_{2}$. From Remark 2, we know that $Q_{c, 1}^{*} \leq \bar{Q}_{c}^{*}$. Therefore, $\bar{Q}_{d}^{*}<Q_{c, 1}^{*} \leq \bar{Q}_{c}^{*}$ which implies $\alpha_{1}<Q_{c, 1}^{*}<\alpha_{2}$. Hence, $F\left(Q_{c, 1}^{*}\right) \neq 0$ or $F\left(Q_{c, 1}^{*}\right) \neq 1$.

Since $Q_{c, 1}^{*} \leq \bar{Q}_{c}^{*}$, we have $F\left(Q_{c, 1}^{*}\right) \leq F\left(\bar{Q}_{c}^{*}\right)$. Under the new pricing of the vendor, the unit price is $c_{1}=c-(r+b-v)\left[F\left(Q_{c, 1}^{*}\right)-F\left(\bar{Q}_{d}^{*}\right)\right]$. Substituting $(r+b-c) /(r+b-v)$ for the value of $F\left(\bar{Q}_{d}^{*}\right)$, we obtain $c_{1}=r+b-(r+b-v) F\left(Q_{c, 1}^{*}\right)$. Since $F\left(Q_{c, 1}^{*}\right) \leq F\left(\bar{Q}_{c}^{*}\right)$, we have $c_{1}>r+b-(r+b-v) F\left(\bar{Q}_{c}^{*}\right)$. Recall from Eq. (5) that $F\left(\bar{Q}_{c}^{*}\right)=(r+b-$ $p) /(r+b-v)$. Therefore, $c_{1}>p$ and, hence, $c_{1}>v$ (if $c<v$, then the buyer would buy an infinite amount). Under the unit price $c_{1}$, using Eq. (3), it can be shown that $\bar{Q}_{d}^{*}\left(c_{1}\right)=Q_{c, 1}^{*}$. Therefore, the buyer is motivated to order $Q_{c, 1}^{*}$ units.

From Proposition 5, we have that $\bar{\Pi}_{b}\left(Q_{c, 1}^{*}, c_{1}\right)>\bar{\Pi}_{b}\left(\bar{Q}_{d}^{*}, c\right)$. If the buyer is asked to pay a fee of $\bar{\Pi}_{b}\left(Q_{c, 1}^{*}, c_{1}\right)-\bar{\Pi}_{b}\left(\bar{Q}_{d}^{*}, c\right)$, then his/her total expected profit is $\bar{\Pi}_{b}\left(Q_{c, 1}^{*}, c_{1}\right)-\bar{\Pi}_{b}\left(Q_{c, 1}^{*}, c_{1}\right)+\bar{\Pi}_{b}\left(\bar{Q}_{d}^{*}, c\right)=\bar{\Pi}_{b}\left(\bar{Q}_{d}^{*}, c\right)$.

PROOF OF PROPOSITION 7: Noting that $c_{2}=c+(r+b-v)\left[F\left(\bar{Q}_{d}^{*}\right)-\right.$ $F\left(Q_{c, 1}^{*}\right)$ ], and using Expression (3), we obtain $c_{2}=r+b-(r+b-$ v) $F\left(Q_{c, 1}^{*}\right)$. Again using Eq. (3), we conclude that the buyer can be motivated to order $Q_{c, 1}^{*}$ units under this new price schedule if the vendor pays the buyer a fee of $\bar{\Pi}_{b}\left(\bar{Q}_{d}^{*}, c\right)-\bar{\Pi}_{b}\left(Q_{c, 1}^{*}, c_{2}\right)$. This is simply because, from Proposition 5 , we have $\bar{\Pi}_{b}\left(\bar{Q}_{d}^{*}, c\right)>\bar{\Pi}_{b}\left(Q_{c, 1}^{*}, c_{2}\right)$ so the buyer's resulting expected total profit is $\bar{\Pi}_{b}\left(\bar{Q}_{d}^{*}, c\right)-\bar{\Pi}_{b}\left(Q_{c, 1}^{*}, c_{2}\right)+\bar{\Pi}_{b}\left(Q_{c, 1}^{*}, c_{2}\right)=\bar{\Pi}_{b}\left(\bar{Q}_{d}^{*}, c\right)$. 
PROOF OF PROPOSITION 8: First, we will show that $\bar{\Pi}_{b}\left(Q_{c, 1}^{*}, c_{3}\right)=$ $\bar{\Pi}_{b}\left(\bar{Q}_{d}^{*}, c\right)$. Note that, by definition,

$$
\begin{aligned}
c_{3} & =c-\frac{\bar{\Pi}_{b}\left(\bar{Q}_{d}^{*}, c\right)-\bar{\Pi}_{b}\left(Q_{c, 1}^{*}, c\right)}{Q_{c, 1}^{*}}, \text { and } \bar{\Pi}_{b}(Q, c) \\
& =(-c+v) Q-K_{b}+(r-v) E[X]-(r-v+b) \int_{Q}^{\infty}(x-Q) f(x) d x .
\end{aligned}
$$

As a consequence, we can write

$$
\begin{aligned}
\bar{\Pi}_{b}\left(Q_{c, 1}^{*}, c_{3}\right)=( & \left.-c+\frac{\bar{\Pi}_{b}\left(\bar{Q}_{d}^{*}, c\right)-\bar{\Pi}_{b}\left(Q_{c, 1}^{*}, c\right)}{Q_{c, 1}^{*}}+v\right) Q_{c, 1}^{*}-K_{b} \\
& +(r-v) E[X]-(r-v+b) \int_{Q_{c, 1}^{*}}^{\infty}\left(x-Q_{c, 1}^{*}\right) f(x) d x,
\end{aligned}
$$

which in turn results in

$$
\begin{aligned}
\bar{\Pi}_{b}\left(Q_{c, 1}^{*}, c_{3}\right)= & \bar{\Pi}_{b}\left(\bar{Q}_{d}^{*}, c\right)-\bar{\Pi}_{b}\left(Q_{c, 1}^{*}, c\right)+(-c+v) Q_{c, 1}^{*}-K_{b} \\
& +(r-v) E[X]-(r-v+b) \int_{Q_{c, 1}^{*}}^{\infty}\left(x-Q_{c, 1}^{*}\right) f(x) d x .
\end{aligned}
$$

Substituting the expression of $\bar{\Pi}_{b}\left(Q_{c, 1}^{*}, c\right)$, the above equation leads to $\bar{\Pi}_{b}\left(Q_{c, 1}^{*}, c_{3}\right)=\bar{\Pi}_{b}\left(\bar{Q}_{d}^{*}, c\right)$.

In order to prove that $Q_{c, 1}^{*}$ maximizes the buyer's expected profit function, first we show that $\bar{Q}_{d}^{*}<\bar{Q}_{d}^{*}\left(c_{3}\right)<Q_{c, 1}^{*}$. Since $c_{3}<c$, it follows from Expression (3) that $\bar{Q}_{d}^{*}<\bar{Q}_{d}^{*}\left(c_{3}\right)$. Note that $\bar{\Pi}_{b}\left(\bar{Q}_{d}^{*}, c_{3}\right)>\bar{\Pi}_{b}\left(\bar{Q}_{d}^{*}, c\right)$ because $c_{3}<c$ and $\bar{\Pi}_{b}(Q, c)$ is decreasing in $c$ for fixed values of $Q$. Since $\bar{\Pi}_{b}\left(Q_{c, 1}^{*}, c_{3}\right)=\bar{\Pi}_{b}\left(\bar{Q}_{d}^{*}, c\right)$, we also have $\bar{\Pi}_{b}\left(\bar{Q}_{d}^{*}, c_{3}\right)>\bar{\Pi}_{b}\left(Q_{c, 1}^{*}, c_{3}\right)$. Recall that for a fixed value of $c, \bar{\Pi}_{b}(Q, c)$ is a strictly concave function of $Q$. Therefore, if $Q_{c, 1}^{*}>\bar{Q}_{d}^{*}$ and $\bar{Q}_{d}^{*}<\bar{Q}_{d}^{*}\left(c_{3}\right)$, then $\bar{\Pi}_{b}\left(\bar{Q}_{d}^{*}, c_{3}\right)>$ $\bar{\Pi}_{b}\left(Q_{c, 1}^{*}, c_{3}\right)$ is true only if $\bar{Q}_{d}^{*}<\bar{Q}_{d}^{*}\left(c_{3}\right)<Q_{c, 1}^{*}$. It follows that $\bar{\Pi}_{b}\left(Q, c_{3}\right)<\bar{\Pi}_{b}\left(Q_{c, 1}^{*}, c_{3}\right), \forall Q>Q_{c, 1}^{*}$. Since $\bar{Q}_{d}^{*}$ maximizes $\bar{\Pi}_{b}(Q, c)$, we have $\bar{\Pi}_{b}(Q, c)<\bar{\Pi}_{b}\left(\bar{Q}_{d}^{*}, c\right)=\bar{\Pi}_{b}\left(Q_{c, 1}^{*}, c_{3}\right), \forall Q<Q_{c, 1}^{*}$, and $Q \neq \bar{Q}_{d}^{*}$. Therefore, $Q_{c, 1}^{*}$ maximizes $\bar{\Pi}_{b}\left(Q, c_{3}\right)$ under the new pricing schedule.

PROOF OF PROPOSITION 9: Similar to the proof of Proposition 8, it can be easily shown that $\bar{\Pi}_{b}\left(Q_{c, 1}^{*}, c_{4}\right)=\bar{\Pi}_{b}\left(\bar{Q}_{d}^{*}, c\right)$. Since $c_{4}<c$, it follows from Expression (3) that $\bar{Q}_{d}^{*}<\bar{Q}_{d}^{*}\left(c_{4}\right)$. Since $Q_{c, 1}^{*}<\bar{Q}_{d}^{*}$, we have $\bar{Q}_{d}^{*}\left(c_{4}\right)>\bar{Q}_{d}^{*}>Q_{c, 1}^{*}$. Recall that $\bar{\Pi}_{b}\left(Q, c_{4}\right)$ is a strictly concave function of $Q$ and $\bar{Q}_{d}^{*}\left(c_{4}\right)$ is its unique maximizer. It follows that $\bar{\Pi}_{b}\left(Q_{c, 1}^{*}, c_{4}\right)>\bar{\Pi}_{b}\left(Q, c_{4}\right), \forall Q<Q_{c, 1}^{*}$. For $Q>Q_{c, 1}^{*}$ and $Q \neq \bar{Q}_{d}^{*}$, we have $\bar{\Pi}_{b}\left(Q_{c, 1}^{*}, c_{4}\right)=\bar{\Pi}_{b}\left(\bar{Q}_{d}^{*}, c\right)>\bar{\Pi}_{b}(Q, c)$. Therefore, $Q_{c, 1}^{*}$ maximizes $\bar{\Pi}_{b}\left(Q, c_{4}\right)$ under the new pricing schedule.

\section{A.6. Properties of $\Pi_{c}^{I I}(Q)$}

PROOF OF PROPERTY 10: Since $\bar{\Pi}_{c}(Q)$ is a strictly concave function of $Q$ and $\bar{Q}_{c}^{*}$ is its maximizer, $\bar{\Pi}_{c}(Q)$ is decreasing $\forall Q>\bar{Q}_{c}^{*}$. Observe that $\left(-\left\lceil Q / P_{v}\right\rceil R_{v}\right)$ and $\left(-\left\lceil Q / P_{b}\right\rceil R_{b}\right)$ are non-increasing functions. The sum of a decreasing and a non-increasing function is decreasing. Therefore, $\Pi_{c}^{I I}(Q)$ is decreasing $\forall Q>\bar{Q}_{c}^{*}$.

PROOF OF PROPERTY 11: Since $\bar{\Pi}_{c}(Q)$ is strictly concave with a maximizer at $\bar{Q}_{c}^{*}, \bar{\Pi}_{c}\left(Q_{1}\right)<\bar{\Pi}_{c}\left(Q_{2}\right), \forall Q_{1}, Q_{2}$ s.t. $Q_{1}<Q_{2} \leq \bar{Q}_{c}^{*}$. When $\left(k_{1}-1\right) P_{b}<Q_{1}<Q_{2} \leq k_{1} P_{b}$, we have $\left\lceil Q_{1} / P_{b}\right\rceil=\left\lceil Q_{2} / P_{b}\right\rceil=k_{1}$. Similarly, when $\left(k_{2}-1\right) P_{v}<Q_{1}<Q_{2} \leq k_{2} P_{v}$, we have $\left\lceil Q_{1} / P_{v}\right\rceil=$ $\left\lceil Q_{2} / P_{v}\right\rceil=k_{2}$. Therefore, $\bar{\Pi}_{c}\left(Q_{1}\right)-\left\lceil Q_{1} / P_{v}\right\rceil R_{v}-\left\lceil Q_{1} / P_{b}\right\rceil R_{b}<$ $\bar{\Pi}_{c}\left(Q_{2}\right)-\left\lceil Q_{2} / P_{v}\right\rceil R_{v}-\left\lceil Q_{2} / P_{b}\right\rceil R_{b}$ so that $\Pi_{c}^{I I}\left(Q_{1}\right)<\Pi_{c}^{I I}\left(Q_{2}\right)$.

\section{A.7. Coordinated Solutions for Model II/ Vendor-Managed Incentive and Delivery Contracts: Proofs}

PROOF OF PROPOSITION 10: Note that in both cases the buyer is compensated with a fixed payment of $\Pi_{b}\left(Q_{d, 2}^{*}\right)-\Pi_{b}\left(Q_{c, 2}^{*}\right)$. Therefore, if he/she orders $Q_{c, 2}^{*}$, then he/she stays at a no worse expected profit value, which is $\Pi_{b}\left(Q_{d, 2}^{*}\right)-\Pi_{b}\left(Q_{c, 2}^{*}\right)+\Pi_{b}\left(Q_{c, 2}^{*}\right)=\Pi_{b}\left(Q_{d, 2}^{*}\right)$. Next, we show that in each case of the proposition the maximum attainable expected profit value for the buyer is not greater than $\Pi_{b}\left(Q_{d, 2}^{*}\right)$.

For the first part (i.e., $Q_{c, 2}^{*}>\bar{Q}_{d}^{*}$ ), we use the fact that $\Pi_{b}(Q)$ is decreasing after $\bar{Q}_{d}^{*}$. That is, $\Pi_{b}\left(Q_{2}\right)<\Pi_{b}\left(Q_{1}\right)$ for all $Q_{1}$ and $Q_{2}$ such that $\bar{Q}_{d}^{*} \leq Q_{1}<Q_{2}$. Adding a fixed value of $\Pi_{b}\left(Q_{d, 2}^{*}\right)-\Pi_{b}\left(Q_{c, 2}^{*}\right)$ to both sides of this inequality results in $\Pi_{b}\left(Q_{2}\right)+\Pi_{b}\left(Q_{d, 2}^{*}\right)-\Pi_{b}\left(Q_{c, 2}^{*}\right)<$ $\Pi_{b}\left(Q_{1}\right)+\Pi_{b}\left(Q_{d, 2}^{*}\right)-\Pi_{b}\left(Q_{c, 2}^{*}\right)$, which implies that the buyer's expected profit function is still decreasing after $\bar{Q}_{d}^{*}$ under the new pricing strategy. Therefore, the maximum value of the buyer's expected profit in this region is realized at the smallest value of $Q$, which is $Q_{c, 2}^{*}$.

For the second part (i.e., $Q_{c, 2}^{*}<\bar{Q}_{d}^{*}$ ), we use Property 2, which implies that to the left of $\bar{Q}_{d}^{*}, \Pi_{b}(Q)$ is piecewise increasing. Adding a fixed value to each piece does not change the fact that $\Pi_{b}(Q)$ is increasing over $\left(\left(\left\lceil Q_{c, 2}^{*} / P_{b}\right\rceil-1\right) P_{b}, Q_{c, 2}^{*}\right]$. Therefore, under the new pricing strategy, $Q_{c, 2}^{*}$ maximizes the buyer's expected profit.

PROOF OF PROPOSITION 11: From Expression (13), we have $Q_{d, 2}^{*} \leq$ $\bar{Q}_{d}^{*}$. We also know from Eqs. (3) and (5) that $\bar{Q}_{d}^{*} \leq \bar{Q}_{c}^{*}$. Therefore, $Q_{d, 2}^{*} \leq$ $\bar{Q}_{d}^{*} \leq \bar{Q}_{c}^{*}$, which implies that $Q_{c, 2}^{*}<\bar{Q}_{d}^{*}$ and $Q_{c, 2}^{*}<\bar{Q}_{c}^{*}$. As a result, $Q_{c, 2}^{*}$ can either be an integer multiple of $P_{b}$ or $P_{v}$. In both cases, if the buyer is compensated with a fixed payment of $\Pi_{b}\left(Q_{d, 2}^{*}\right)-\Pi_{b}\left(Q_{c, 2}^{*}\right)$ and orders $Q_{c, 2}^{*}$ units, then he/she again stays at a no worse expected profit level, which is $\Pi_{b}\left(Q_{d, 2}^{*}\right)-\Pi_{b}\left(Q_{c, 2}^{*}\right)+\Pi_{b}\left(Q_{c, 2}^{*}\right)=\Pi_{b}\left(Q_{d, 2}^{*}\right)$. However, in order to complete the proof, we need to show that the maximum attainable expected profit value for the buyer is not greater than $\Pi_{b}\left(Q_{d, 2}^{*}\right)$.

From Property 2, we know that for $Q<\bar{Q}_{d}^{*}, \Pi_{b}(Q)$ is piecewise increasing. Since $Q_{c, 2}^{*}<Q_{d, 2}^{*}$, we can also conclude that before $Q_{c, 2}^{*}, \Pi_{b}(Q)$ is increasing at integer multiples of $P_{b}$. Otherwise, from Proposition 2, $Q_{d, 2}^{*}$ would take a value that is less than $Q_{c, 2}^{*}$. Thus, for the first part of the proposition, we show that the buyer's expected profit function in the given region (i.e., $\left.\left(-\infty, Q_{c, 2}^{*}\right]\right)$ reaches its maximum at $Q_{c, 2}^{*}$. The second part of the proof follows from the fact that $\Pi_{b}(Q)$ is piecewise increasing.

PROOF OF PROPOSITION 12: In order to show that the proposed contract is feasible, we prove the following:

$$
\begin{aligned}
& \text { - } \alpha_{1}<Q_{c, V M D}^{*}<\alpha_{2} \text { where } \alpha_{1}=\inf \{x: f(x)>0\} \text { and } \alpha_{2}= \\
& \quad \sup _{2}\{x: f(x)>0\}, \text { and } \\
& \text { - } \bar{\Pi}_{b}\left(Q_{c, V M D}^{*}, c_{5}\right)>\Pi_{b}\left(Q_{d, 2}^{*}, c\right) \text {. }
\end{aligned}
$$

Observe that function $\Pi_{c}^{V M D}(Q)$ in Expression (15) has the same characteristics as function $\Pi_{c}^{I I}(Q)$ in Expression (14). Therefore, from Property 10, we have $Q_{c, V M D}^{*} \leq \bar{Q}_{c}^{*}$. Also, by definition, we have $\alpha_{1}<\bar{Q}_{d}^{*}<\alpha_{2}$ and $\alpha_{1}<\bar{Q}_{c}^{*}<\alpha_{2}$. Hence, using the fact that $\bar{Q}_{c}^{*} \geq Q_{c, V M D}^{*} \geq \bar{Q}_{d}^{*}$, we can write $\alpha_{1}<Q_{c, V M D}^{*}<\alpha_{2}$ so that $0<F\left(Q_{c, V M D}^{*}\right)<1$, and this proves the first item in the above list.

By definition, $\bar{\Pi}_{b}(Q, c)>\Pi_{b}(Q, c), \forall Q \geq 0$, so that we can write $\bar{\Pi}_{b}\left(Q_{d, 2}^{*}, c\right)>\Pi_{b}\left(Q_{d, 2}^{*}, c\right)$. Furthermore, since $\bar{Q}_{d}^{*}$ is the maximizer of $\bar{\Pi}_{b}(Q, c)$, we have $\bar{\Pi}_{b}\left(\bar{Q}_{d}^{*}, c\right)>\bar{\Pi}_{b}\left(Q_{d, 2}^{*}, c\right)>\Pi_{b}\left(Q_{d, 2}^{*}, c\right)$. Recalling that $c_{5}<c$, we also have $\bar{\Pi}_{b}\left(Q, c_{5}\right)>\bar{\Pi}_{b}(Q, c), \forall Q \geq 0$. As a result,

$$
\bar{\Pi}_{b}\left(\bar{Q}_{d}^{*}, c_{5}\right)>\bar{\Pi}_{b}\left(\bar{Q}_{d}^{*}, c\right)>\bar{\Pi}_{b}\left(Q_{d, 2}^{*}, c\right)>\Pi_{b}\left(Q_{d, 2}^{*}, c\right) .
$$

Note that under the new unit price $c_{5}$ the buyer's expected profit function is given by $\bar{\Pi}_{b}\left(Q, c_{5}\right)$. Hence, in order to complete the feasibility proof, it 
suffices to show that $Q_{c, V M D}^{*}$ is the maximizer of $\bar{\Pi}_{b}\left(Q, c_{5}\right)$ so that

$$
\bar{\Pi}_{b}\left(Q_{c, V M D}^{*}, c_{5}\right)>\bar{\Pi}_{b}\left(\bar{Q}_{d}^{*}, c_{5}\right) .
$$

Combining Expressions (22) and (23), we have $\bar{\Pi}_{b}\left(Q_{c, V M D}^{*}, c_{5}\right)>$ $\Pi_{b}\left(Q_{d, 2}^{*}, c\right)$.

Recalling Expressions (2) and (3), and utilizing the expression of $c_{5}$ given in Proposition 12, it can be easily shown that the maximizer of $\bar{\Pi}_{b}\left(Q, c_{5}\right)$ is given by the $Q$ value where

$$
F(Q)=\frac{r+b-\left[c-(r+b-v) F\left(Q_{c, V M D}^{*}\right)+(r+b-v) F\left(\bar{Q}_{d}^{*}\right)\right]}{r+b-v} .
$$

After a little algebra, the right-hand side of the above equation reduces to $F\left(Q_{c, V M D}^{*}\right)$, and, hence, $Q_{c, V M D}^{*}$ maximizes $\bar{\Pi}_{b}\left(Q, c_{5}\right)$ so that $\bar{\Pi}_{b}\left(Q_{c, V M D}^{*}, c_{5}\right)>\Pi_{b}\left(Q_{d, 2}^{*}, c\right)$, and this completes the feasibility proof.

We continue with showing that under the proposed contract $Q_{c, V M D}^{*}$ also maximizes the buyer's decentralized expected profits, and the buyer is still expected earn $\Pi_{b}\left(Q_{d, 2}^{*}, c\right)$ by ordering $Q_{c, V M D}^{*}$ units. Note that, under the proposed contract, the buyer's expected profit function can be expressed as

$$
\Pi_{b}^{V M D}(Q)=\bar{\Pi}_{b}\left(Q, c_{5}\right)-\left[\bar{\Pi}_{b}\left(Q_{c, V M D}^{*}, c_{5}\right)-\Pi_{b}\left(Q_{d, 2}^{*}, c\right)\right] .
$$

Since the second term of the above function is a fixed value, the maximizer of $\Pi_{b}^{V M D}(Q)$ is the same as the maximizer of $\bar{\Pi}_{b}\left(Q, c_{5}\right)$, and it is given by $Q_{c, V M D}^{*}$. Consequently, under the proposed contract, the buyer will order $Q_{c, V M D}^{*}$ units leading to $\Pi_{b}^{V M D}\left(Q_{c, V M D}^{*}\right)=\Pi_{b}\left(Q_{d, 2}^{*}, c\right)$.

PROOF OF PROPOSITION 13: The proposed contract is feasible because, by assumption, we have $\alpha_{1}<Q_{c, V M D}^{*}$ and $Q_{c, V M D}^{*}<\bar{Q}_{d}^{*}$ so that $\alpha_{1}<Q_{c, V M D}^{*}<\alpha_{2}$. In order to complete the proof, we proceed with showing that, under the proposed contract, $Q_{c, V M D}^{*}$ maximizes the buyer's decentralized expected profit function so that the buyer is still expected to earn $\Pi_{b}\left(Q_{d, 2}^{*}, c\right)$ by ordering $Q_{c, V M D}^{*}$ units.

Observe that under the new unit price buyer's expected profits may be more or less than $\Pi_{b}\left(Q_{d, 2}^{*}, c\right)$. However, his/her final expected profits may be adjusted by fixed payments. The buyer or the vendor may make a fixed payment to the other party depending on whether $\bar{\Pi}_{b}\left(Q_{c, V M D}^{*}, c_{6}\right)>$ $\Pi_{b}\left(Q_{d, 2}^{*}, c\right)$ or $\bar{\Pi}_{b}\left(Q_{c, V M D}^{*}, c_{6}\right)<\Pi_{b}\left(Q_{d, 2}^{*}, c\right)$. In either case, the buyer's expected profit function can be represented by

$$
\Pi_{b}^{V M D}(Q)=\bar{\Pi}_{b}\left(Q, c_{6}\right)-\left[\bar{\Pi}_{b}\left(Q_{c, V M D}^{*}, c_{6}\right)-\Pi_{b}\left(Q_{d, 2}^{*}, c\right)\right] .
$$

Note that if $\bar{\Pi}_{b}\left(Q_{c, V M D}^{*}, c_{6}\right)>\Pi_{b}\left(Q_{d, 2}^{*}, c\right)$, then $\bar{\Pi}_{b}\left(Q_{c, V M D}^{*}, c_{6}\right)-$ $\Pi_{b}\left(Q_{d, 2}^{*}, c\right)$ is positive and the adjustment is aimed at decreasing the buyer's expected profits. If $\bar{\Pi}_{b}\left(Q_{c, V M D}^{*}, c_{6}\right)<\Pi_{b}\left(Q_{d, 2}^{*}, c\right)$, then $\bar{\Pi}_{b}\left(Q_{c, V M D}^{*}, c_{6}\right)-$ $\Pi_{b}\left(Q_{d, 2}^{*}, c\right)$ is negative and the adjustment is aimed at increasing the buyer's expected profits.

In order to show that $Q_{c, V M D}^{*}$ is the maximizer of $\bar{\Pi}_{b}\left(Q, c_{6}\right)$, let us recall Expressions (2) and (3) and utilize the expression of $c_{6}$ given in Proposition 13 . It can be easily shown that the maximizer of $\bar{\Pi}_{b}\left(Q, c_{6}\right)$ is given by the $Q$ value where

$$
\begin{array}{r}
F(Q)=\frac{r+b-\left[c+(r+b-v) F\left(\bar{Q}_{d}^{*}\right)-(r+b-v) F\left(Q_{c, V M D}^{*}\right)\right]}{r+b-v}=F\left(Q_{c, V M D}^{*}\right) .
\end{array}
$$

It follows that, $Q_{c, V M D}^{*}$ maximizes $\bar{\Pi}_{b}\left(Q, c_{6}\right)$. Under the proposed contract, the buyer's expected profit function $\Pi_{b}^{V M D}(Q)$ is given by

$$
\bar{\Pi}_{b}\left(Q, c_{6}\right)-\left[\bar{\Pi}_{b}\left(Q_{c, V M D}^{*}, c_{6}\right)-\Pi_{b}\left(Q_{d, 2}^{*}, c\right)\right] .
$$

Naval Research Logistics DOI 10.1002/nav
Since the second term of the above expression is a fixed value, the maximizer of $\Pi_{b}^{V M D}(Q)$ is the same as the maximizer of $\bar{\Pi}_{b}\left(Q, c_{6}\right)$, and it is given by $Q_{c, V M D}^{*}$. Consequently, under the proposed contract, the buyer will order $Q_{c, V M D}^{*}$ units leading to $\Pi_{b}^{V M D}\left(Q_{c, V M D}^{*}\right)=\Pi_{b}\left(Q_{d, 2}^{*}, c\right)$, and this completes the proof.

\section{PROOF OF PROPOSITION 14:}

- Case 1. Since $P_{b}^{V M D} \geq P_{b}$, we have $\left\lceil Q / P_{b}^{V M D}\right\rceil \leq\left\lceil Q / P_{b}\right\rceil, \forall Q \geq 0$. Also, since $R_{b}^{V M D} \leq R_{b}$, we further have $\left\lceil Q / P_{b}^{V M D}\right\rceil R_{b}^{V M D} \leq$ $\left\lceil Q / P_{b}\right\rceil R_{b}, \forall Q \geq 0$. Then, using Expressions (14) and (15), we have

$$
\begin{aligned}
\Pi_{c}^{I I}(Q)=\bar{\Pi}_{c}(Q)-\left\lceil\frac{Q}{P_{v}}\right\rceil R_{v}-\left\lceil\frac{Q}{P_{b}}\right\rceil R_{b} \leq \Pi_{c}^{V M D}(Q) \\
=\bar{\Pi}_{c}(Q)-\left\lceil\frac{Q}{P_{v}}\right\rceil R_{v}-\left\lceil\frac{Q}{P_{b}^{V M D}}\right\rceil R_{b}^{V M D}, \forall Q \geq 0,
\end{aligned}
$$

and, thus, $\max _{Q \geq 0} \Pi_{c}^{I I}(Q) \leq \max _{Q \geq 0} \Pi_{c}^{V M D}(Q)$.

- Case 2. Since $P_{b}^{\overline{V M D}} \leq P_{b}$, we have $\left\lceil Q / P_{b}^{V M D}\right\rceil \geq\left\lceil Q / P_{b}\right\rceil, \forall Q \geq$ 0 . Also, since $R_{b}^{V M D} \geq R_{b}$, we have $\left\lceil Q / P_{b}^{V M D}\right\rceil R_{b}^{V M D} \geq$ $\left\lceil Q / P_{b}\right\rceil R_{b}, \forall Q \geq 0$. Using Expressions (14) and (15), this in turn implies that $\Pi_{c}^{I I}(Q) \geq \Pi_{c}^{V M D}(Q), \forall Q \geq 0$, and, thus, $\max _{Q \geq 0} \Pi_{c}^{I I}(Q) \geq \max _{Q \geq 0} \Pi_{c}^{V M D}(Q)$.

- Case 3. Suppose that $P_{b}^{V M D} \geq P_{b}<\infty$ and let $R_{b}^{V M D} \rightarrow \infty$, and $R_{b} \rightarrow 0$. Under these assumptions, $\left\lceil Q / P_{b}^{V M D}\right\rceil R_{b}^{V M D}>$ $\left\lceil Q / P_{b}\right\rceil R_{b}, \forall Q \geq 0$, and it follows from Expressions (14) and (15) that $\Pi_{c}^{I I}(Q)>\Pi_{c}^{V M D}(Q), \forall Q \geq 0$. Hence, $\max _{Q \geq 0} \Pi_{c}^{I I}(Q)>$ $\max _{Q \geq 0} \Pi_{c}^{V M D}(Q)$.

Now, suppose that $\infty>R_{b}^{V M D}>R_{b}$, and let $P_{b}^{V M D} \rightarrow \infty$ and $P_{b} \rightarrow 0$. Under these assumptions, $\left\lceil Q / P_{b}^{V M D}\right\rceil R_{b}^{V M D}<$ $\left\lceil Q / P_{b}\right\rceil R_{b}, \forall Q \geq 0$, and, again, Expressions (14) and (15) imply $\Pi_{c}^{I I}(Q)<\Pi_{c}^{V M D}(Q), \forall Q \geq 0$, so that $\max _{Q \geq 0} \Pi_{c}^{I I}(Q)<$ $\max _{Q \geq 0} \Pi_{c}^{V M D}(Q)$.

- Case 4. The proof for Case 3 can be easily extended for this case.

\section{REFERENCES}

[1] O. Alp, N. Erkip, and R. Güllü, Optimal lot sizing/vehicle dispatching policies under stochastic lead times and stepwise fixed costs, Oper Res 51(1) (2003), 160-171.

[2] A. Banerjee, On a quantity discount pricing model to increase vendor profits, Manage Sci 32 (1986), 1513-1517.

[3] T. Boyac1 and G. Gallego, Cordinating pricing and inventory replenishment policies for one wholesaler and one or more geographically dispersed retailers, Int J Prod Econ 77 (2002), 95-111.

[4] G.P. Cachon and M. Fisher, Supply chain inventory management and the value of shared information, Manage Sci 46 (2000), 1032-1048.

[5] L.M.A. Chan, A. Muriel, Z.-J. Shen, D. Simchi-Levi, and C.-P. Teo, Effective zero inventory ordering polices for the singlewarehouse multi-retailer problem with piecewise linear cost structures, Manage Sci 48 (2002), 1446-1460.

[6] S. Çetinkaya and C.-Y. Lee, Stock replenishment and shipment scheduling for vendor managed inventory systems, Manage Sci 46(2) (2000), 217-232.

[7] K.L. Cheung and H.L. Lee, The inventory benefit of shipment coordination and stock rebalancing in a supply chain, Manage Sci 48(2) (2002), 300-306.

[8] C.J. Corbett and X. Groote, A supplier's optimal quantity discount policy under asymmetric information, Manage Sci 46 (2000), 444-450. 
[9] S.M. Disney, A.T. Potter, and B.M. Gardner, The impact of vendor managed inventory on transport operations, Transport Res E 39 (2003), 363-380.

[10] S.M. Disney and D.R. Towill, The effect of vendor managed inventory (VMI) dynamics on the bullwhip effect in supply chains, Int J Prod Econ 85 (2003), 199-215.

[11] Y. Dong and K. Xu, A supply chain model of vendor managed inventory, Transport Res E 38 (2003), 75-95.

[12] H. Emmons and S.M. Gilbert, The role of returns policies in pricing and inventory decisions for catalogue goods, Manage Sci 44 (1998), 276-283.

[13] Y. Gerchak and Y. Wang, Revenue sharing vs. wholesale-price contracts in assembly systems with random demand, Prod Oper Manage 13(1) (2004), 23-33.

[14] S.K. Goyal, An integrated inventory model for a singlesupplier single-customer problem, Int J Prod Res 15 (1976), 107-111.

[15] A. Ha, Supplier-buyer contracting: Asymmetric cost information and cutoff level policy for buyer participation, Nav Res Logist 48 (2001), 41-64.

[16] C. Hofmann, Supplier's pricing policy in a just-in-time environment, Comput Oper Res 27 (2000), 1357-1373.

[17] M.A. Hoque and S.K. Goyal, An optimal policy for singlevendor single-buyer integrated production-inventory system with capacity constraint of transport equipment, Int J Prod Econ 65 (2000), 305-315.

[18] A.P. Jeuland and S.M. Shugan, Managing channel profits, Market Sci 2 (1983), 239-272.

[19] R. Kaipia, J. Holmström, and K. Tanskanen, VMI: What are you loosing if you let your customer place orders? Prod Plan Contr 13(1) (2002), 17-25.

[20] R. Lal and R. Staelin, An approch for developing an optimal discount pricing policy, Manage Sci 30 (1984), 1524-1539.

[21] C.-Y. Lee, A solution to the multiple setup problem with dynamic demand, IIE Trans 21(3) (1989), 266-270.

[22] H.L. Lee and M.J. Rosenblatt, A generalized quantity discount pricing model to increase supplier's profits, Manage Sci 32 (1986), 1177-1185.
[23] H.L. Lee, K. So, and C.S. Tang, The value of information sharing in a two-level supply chain, Manage Sci 46 (2000), 626-643.

[24] S.A. Lippman, Optimal inventory policy with multiple setup costs, Manage Sci 16 (1969), 118-138.

[25] B.K. Mishra and S. Raghunathan, Retailer- vs. vendormanaged inventory and brand competition, Manage Sci 50(4) (2004), 445-457.

[26] J.P. Monahan, A quantity discount pricing model to increase vendor profits, Manage Sci 30 (1984), 720-726.

[27] B.A. Pasternack, Optimal pricing and returns policies for perishable commodities, Market Sci 4 (1985), 166-176.

[28] R. Piplani and S. Viswanathan, A model for evaluating supplier-owned inventory strategy, Int J Prod Econ 81-82 (2003), 565-571.

[29] L.B. Schwarz, A simple continuous review deterministic onewarehouse $N$-retailer inventory problem, Manage Sci 19 (1973), 555-566.

[30] T.A. Taylor, Channel coordination under price protection, midlife returns, and end-of-life returns in dynamic markets, Manage Sci 47 (2001), 1220-1234.

[31] T.A. Taylor, Supply chain coordination under channel rebates with sales effort effects, Manage Sci 48 (2002), 992-1007.

[32] A. Toptal, S. Çetinkaya, and C.-Y. Lee, The buyer-vendor coordination problem: Modeling inbound and outbound cargo capacity and costs, IIE Trans Logist Schedul 35 (2003), 987-1002.

[33] A. Tsay, S. Nahmias, and N. Agrawal, Modeling supply chain contracts: A review. In S. Tayur, R. Ganeshan, M. Magazine, editors. Quantitative Models for Supply Chain Management, Kluwer Academic, Norwell, MA, 2000, p. 299-330.

[34] Z.K. Weng, Channel coordination and quantity discounts, Manage Sci 41 (1995), 1509-1522.

[35] C.A. Yano and A.M. Newman, Scheduling trains and containers with due dates and dynamic arrivals, Transport Sci 34 (2000), 256-270. 